\title{
Effect of composition and pressure on the shear strength of sodium silicate glasses: An atomic scale simulation study
}

\author{
Gergely Molnár, ${ }^{1, *}$ Patrick Ganster, ${ }^{2}$ and Anne Tanguy ${ }^{1, \dagger}$ \\ ${ }^{1}$ LaMCos, INSA-Lyon, CNRS UMR5259, Université de Lyon, F-69621 Villeurbanne, France \\ ${ }^{2}$ Ecole de Mines de Saint-Étienne, Centre SMS, Laboratoire Georges Friedel CNRS-UMR5307, F-42023 Saint-Éstienne, France
}

(Received 8 November 2016; published 14 April 2017)

\begin{abstract}
The elastoplastic behavior of sodium silicate glasses is studied at different scales as a function of composition and pressure, with the help of quasistatic atomistic simulations. The samples are first compressed and then sheared at constant pressure to calculate yield strength and permanent plastic deformations. Changes occurring in the global response are then compared to the analysis of local plastic rearrangements and strain heterogeneities. It is shown that the plastic response results from the succession of well-identified localized irreversible deformations occurring in a nanometer-size area. The size and the number of these local rearrangements, as well as the amount of internal deviatoric and volumetric plastic deformation, are sensitive to the composition and to the pressure. In the early stages of the deformation, plastic rearrangements are driven by sodium mobility. Consequently, the elastic yield strength decreases when the sodium content increases, and the same when pressure increases. Finally, good correlation was found between global and local stress-strain relationships, reinforcing again the role of sodium ions as local initiators of the plastic behavior observed at larger scales.
\end{abstract}

DOI: 10.1103/PhysRevE.95.043001

\section{INTRODUCTION}

Silicate glasses are widely used for their ease in forming and recycling, among other interesting physical and mechanical properties. Sodium silicate glasses are excellent examples of this type of glass with practical interest (hardening accelerator in cements, passive fire protection, etc.). In addition, for theoretical interest, they show a complex mechanical behavior resulting from the mixing of only two different materials: silica $\left(\mathrm{SiO}_{2}\right)$, where $\mathrm{Si}$ atoms acts as network formers, and sodium oxide $\left(\mathrm{Na}_{2} \mathrm{O}\right)$, where $\mathrm{Na}$ acts as network modifiers enhancing the viscosity of the supercooled phase [1]. Sodium silicate glasses are known as "normal glasses," while pure silica glasses have an anomalous mechanical behavior characterized by their anomalous thermal retraction and plastic densification at small scales [2]. Based on the tests done with micrometersize balls at room temperature, Romeis et al. [3] showed that the response of pure silica is dominated by densification. On the other hand, indentation tests performed on soda-lime silica [4,5] showed that the addition of sodium and calcium decreases densification processes but increases shear flow significantly, even below the glass transition temperature [6,7]. Studying the small-scale plastic behavior of silicate glasses is crucial to also understanding crack initiation processes and the brittle behavior of glasses at large scales [8,9]. It is thus very important to have an adequate description of their plastic response at the atomic scale in order to identify and, if possible, to predict their failure behavior. While this kind of description already exists for crystals [10], it is not yet well established in these amorphous glasses, especially due to the lack of experimental measurements at a sufficiently small scale.

In the 1970s, Spaepen [11] and Argon [12] proposed a description of the elementary processes responsible for plasticity in metallic glasses. The description proposed by

\footnotetext{
*gmolnar.work@gmail.com
}

${ }^{\dagger}$ Anne.Tanguy@insa-lyon.fr
Spaepen was based on the existence of randomly distributed soft zones, or free volumes, while the description of Argon described low temperature plasticity as a succession of shear dominated dislocation loops identified as shear transformation zones (STZs). Recently, it was shown that the plastic deformation of amorphous systems can be described as a succession of Eshelby inclusions [13] containing both deviatoric (shear dominated) and compressive (densification) components [14-19]. The description of the number and size of the Eshelby inclusions appearing during plastic deformation in glasses is sufficient to reconstruct the stress-strain curves even at finite shear rates [19-23]. However, actual mesoscopic modeling of plasticity in amorphous materials is based on an evolution equation inspired either from master equation developed from statistical physics [24] or from extremal modeling of self-organized phenomena [25]. Both approaches are written on the spatial and temporal evolution of elementary plastic events (PEs). A realistic description of the mechanical couplings between these objects will of course depend on their actual shape, size, and orientation. Moreover, the succession of local dissipative rearrangements is strongly dependent on the nucleation criteria that are still under debate [26-28]. Finally, larger scale calculations (e.g., finite element calculations [29]) need a general description of the yield surface and plastic flow properties in a general three-dimensional (3D) stress space. It was shown recently that the apparent constitutive laws in silicate glasses have a nontrivial shape depending strongly on the composition and pressure [30]. Current mesoscopic models are neither able to provide such an overall description nor to explain the transition between the anomalous to normal behavior as a function of the composition in silicate glasses [27]. Hence, it is essential for larger scale mechanical modeling of glasses to first describe the composition dependence of the elementary processes responsible for plasticity. Therefore, classical atomistic simulations (molecular dynamics and statics) are an ideal tool to describe these elementary processes and the corresponding yield surface at the micrometer scale [31]. 
To investigate the effect of the composition and pressure on the plastic response of sodium silicates, large scale atomistic simulations were carried out and combined with a detailed description of the elementary processes responsible for plastic flow. First the global response is described throughout hydrostatic and shear tests. Then the change observed in the global shear strength is explained by identifying the aforementioned plastic events and relating them to local stress and strain limits. Finally we show that the overall behavior of the individual events corresponds well with the macroscopically observed phenomena. The paper is organized as follows: in Sec. II, the details of the initial sample generation and the elementary deformation procedure are discussed; in Sec. III, the results obtained at the global scale during hydrostatic and shear deformation are discussed as a function of the composition; in Sec. IV, the elementary processes responsible for plastic deformation are analyzed and a microscopic explanation for the change in plastic response as a function of composition and pressure is proposed; we end the paper with a conclusion and some additional remarks.

\section{METHODS}

\section{A. Atomic sample preparation}

Sodium silicate systems are described using the widely used van Beest-Kramer-van Santen (BKS) potential [32] with the parameters of Yuan and Cormack [33], completed with a repulsive function [34]. As discussed in Ref. [35], within this empirical interatomic interaction potential, each atom supports an effective charge and only repulsive, van der Waals, and effective ionic couplings are taken into account. The polarizability of the electronic cloud, for example, is not taken into account here. However, the results compare reasonably well with the experimental measurements of the mechanical properties. The amorphous glass samples were generated by random sequential placement of the atoms in a cubic simulation box with periodic boundary conditions.

Molecular dynamics [36] in an $N-P-T$ ensemble was performed to equilibrate the liquid at $3000 \mathrm{~K}$ and $0 \mathrm{GPa}$ for 100 ps. The liquids were then quenched to $10^{-5} \mathrm{~K}$ with $10^{+13} \mathrm{~K} / \mathrm{s}$ cooling rate. Four different configurations of each $x \mathrm{Na}_{2} \mathrm{O}-(100-x) \mathrm{SiO}_{2}$ glass system, with $x=5,15$, and $30 \% \mathrm{~mol}$ (referred to as NSx5, NSx15, and NSx30) are generated with 67041 atoms for NSx5, 69849 atoms for NSx15, and 73368 atoms for NSx30. According to the composition, the cutoff in the Buckingham potential was tuned to reproduce the experimental glass densities at ambient pressure after the quench [35]. Using this procedure, the size of the final cubical simulation boxes is $100^{3} \AA^{3}$ for all the samples. At this scale, no finite size effects are expected, as extensively discussed in Refs. [19,35,37]. Structural properties of the generated samples and the effect of sodium is in good accordance with experimental measurements [38-40]. The comparison was already presented in previous studies $[33,35]$.

\section{B. Mechanical deformation}

The generated sodium silicate glass samples are then deformed quasistatically [27,41], using molecular statics simulations [42]. In each deformation step, the shape of the simulation box is modified and the position of the atoms is homogeneously rescaled to describe first homogeneous applied strain $\delta \epsilon$. To find the new equilibrium atomic positions, the Polak-Ribiere conjugate gradient algorithm is then used to reach the nearest local minimum of the potential energy. In order to have a quasielastic response, the maximum elementary applied strain step is set to $(\delta \epsilon)=\left(5 \times 10^{-3}\right) \%$ [35]. Note that viscous and intertial effects are not taken into account here since the dynamics is chosen to follow the closest static equilibrium. In amorphous materials, it was shown that inertial and viscous effects start to play a role at sufficiently large strain rates or temperatures, typically when the damping time becomes larger than the intrinsic vibrational period, as detailed in Ref. [43].

Two types of quasistatic deformations were applied to evaluate the plastic behavior of the samples, and thus the stress response under specific strain conditions. All samples were first submitted to hydrostatic compression and tension to set the proper pressure state. Then pure shear deformation was applied at the desired-constant—-pressure.

The components of the Cauchy stress tensor $(\sigma)$ are calculated using virial stress [44]. Pressure is derived from the diagonal elements: $p=-\operatorname{tr}(\sigma) / 3$. During the initial hydrostatic load, all axial dimensions of the simulation box were changed in order to achieve compaction or extension: no shear strain is applied. The volumetric strain is calculated from the volume variation: $\varepsilon_{V}=d V / V_{0}$, where $d V$ is the volume variation $\left(d V=V-V_{0}\right)$ compared to the initial volume $\left(V_{0}\right)$. In step 2 , the simulation box was tilted in the $x y$ plane to apply shear $\left(\epsilon_{x y}\right)$ at the formerly obtained pressure state. During this stage, the pressure is iteratively controlled by changing the axial size of the simulation box in order to achieve equal axial stresses: $\sigma_{x x}=\sigma_{y y}=\sigma_{z z}=-p$, thus a constant hydrostatic stress state [45]. The maximum value of the pressure and the equivalent shear stress is then registered. The equivalent shear stress $(k)$ can be calculated according to the following equation $[46,47]$ :

$$
\begin{aligned}
k^{2}= & \frac{1}{6}\left[\left(\sigma_{x x}-\sigma_{y y}\right)^{2}+\left(\sigma_{x x}-\sigma_{z z}\right)^{2}+\left(\sigma_{y y}-\sigma_{z z}\right)^{2}\right. \\
& \left.+6\left(\sigma_{x y}^{2}+\sigma_{x z}^{2}+\sigma_{y z}^{2}\right)\right]
\end{aligned}
$$

where $\sigma_{i i}$ corresponds to normal stresses and $\sigma_{i j}$ to shear stresses. In this way, the stress state is decomposed into a hydrostatic component $(p)$ and a pure deviatoric part $(k)$. The same method was used to calculate the equivalent shear strain $\left(\epsilon_{k}\right)$. Only in this case, the Green-Lagrange strain tensor was used instead of the Cauchy stress tensor $[48,49]$.

During step 3, the box is tilted in the opposite direction to reduce the shear stress to zero (keeping the system at the same pressure). Finally, in step 4, the pressure is relaxed to $p=0 \mathrm{GPa}$. The final box shape is compared to the original one to calculate the permanent shear $\left[\epsilon_{x y}^{p l}(p, k)\right]$ and volumetric $\left[\epsilon_{V}^{p l}(p, k)\right]$ strains as a function of the applied stresses. The numerical experiment is carried out for various stress states $(p \in[-14,17] \mathrm{GPa}, k \in[0,8.5] \mathrm{GPa})$ to map the plastic response of the material.

\section{LARGE SCALE MECHANICAL RESPONSE}

In order to describe the plastic response of the material, the results are divided into three separate sections. First, the hydro- 


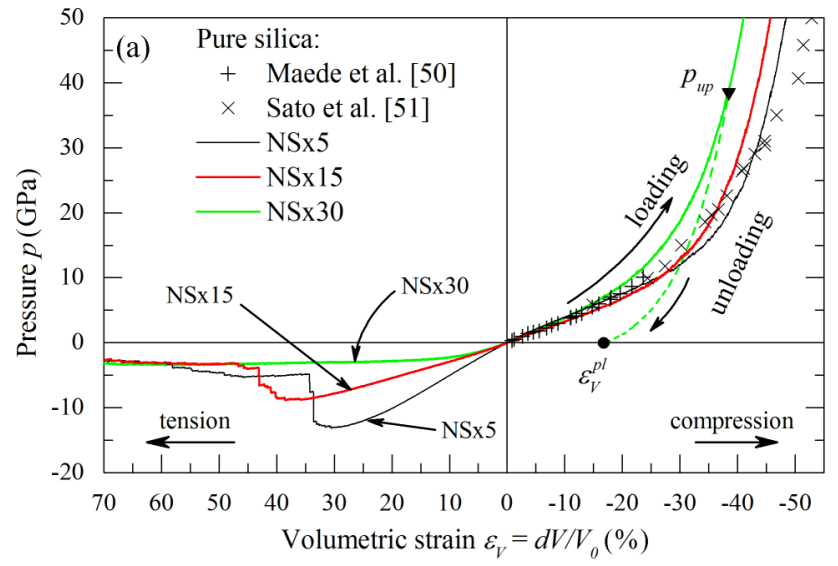

(b) NSx5
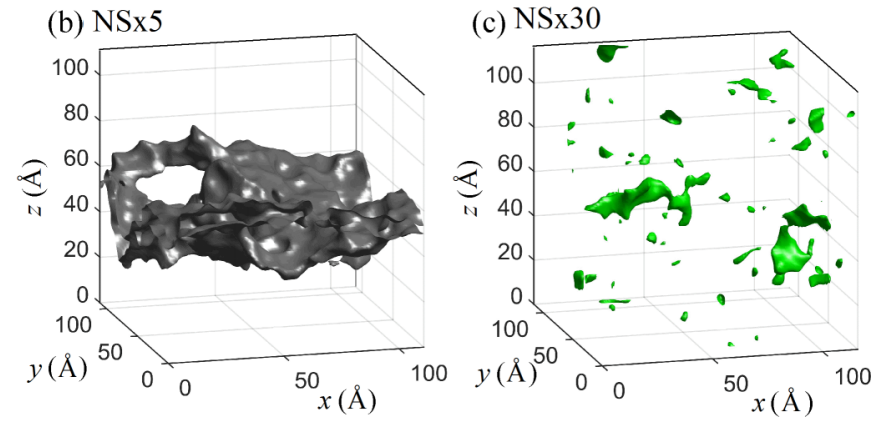

FIG. 1. (a) Pressure as a function of volumetric strain for NSx5, NSx15, and NSx30 glass compositions. The dashed line shows the reverse deformation and the calculated permanent volume change $\left(\epsilon_{V}^{p l}\right)$. In the absence of hydrostatic experiments on sodium silicate, results measured on silica samples are shown [50,51] with cross and plus symbols. (b),(c) Isodensity surfaces $\left(\rho=0.1 \mathrm{~g} / \mathrm{cm}^{3}\right)$ at the minimum pressure state for NSx5 (left) and NSx30 (right).

static response is discussed (without shear deformation). Then several shear cases are studied as a function of composition and pressure. Finally, as explained in Sec. II, the two elementary deformation paths are combined and reversed to map the plastic response as a function of hydrostatic and shear stress.

\section{A. Hydrostatic deformation}

In this section, the results obtained upon pure hydrostatic compression $(k=0 \mathrm{GPa})$ are presented. First the pressure is shown as a function of volumetric strain, then the tangent modulus and the permanent volume change are shown as a function of pressure. Figure 1 shows the pressure as a function of volumetric strain for different compositions (NSx5, $\mathrm{NSx} 15$, and NSx30). The results are compared with different experiments on pure silica [50,51]. The dashed line shows how permanent volumetric strains are computed. All samples are deformed isotropically. After reaching the desired pressure value $\left(p_{u p}\right)$, the direction of the deformation is reversed until the pressure is relaxed to zero. The permanent volume variation is calculated by comparing the initial and final volume size.

Figure 1 shows first that the tensile strength of the material reduces by increasing the sodium content. With more sodium, the material behaves more ductile: after reaching the maximum tensile pressure, the resistance of NSx5 reduces dramatically, while NSx30 maintains its pressure but enters a plastic plateau.

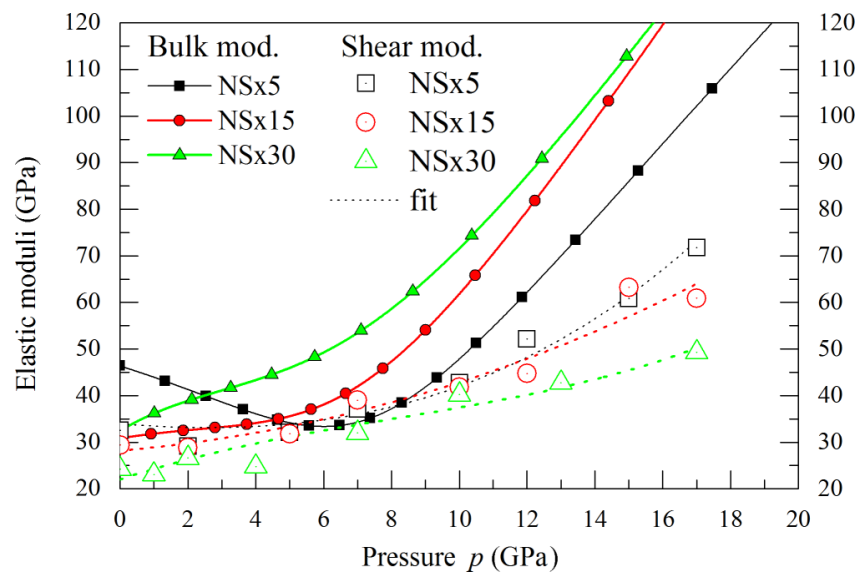

FIG. 2. Pressure dependence of the tangent bulk modulus for NSx5, NSx15, and NSx30 samples. The tangent bulk modulus is calculated by fitting a fifth-order polynomial function on the $p-\epsilon_{V}$ results (shown in Fig. 1) and then the derivative of the fitted curve is taken with respect to $\epsilon_{V}$. The figure also shows the tangent shear modulus with a third-order polynomial, calculated from the shear deformation results (see Fig. 6).

The abrupt change for NSx5 and NSx15 at $\epsilon_{V}=32 \%$ and $40 \%$, respectively, corresponds to stress relaxation caused by discontinuity (crack opening) in the atomic structure. The pressure does not relax to zero at imposed strain due to the nonzero stress components in the in-plane crack directions, as detailed in Ref. [52]. In NSx30, the degradation is much more homogeneous, as seen in Figs. 1(b) and 1(c); therefore, the pressure can be maintained for a long time at a constant plateau. However, after large expansions, the pressure begins to reduce in NSx30 as well, just not in an abrupt way as observed in the other two cases. The compression side of the diagram is different. While on the tensile side, the pressure's amplitude was higher in NSx5 than in the other compositions before breakdown, on the compression side in contrast, the pressure rises quickly in NSx30. Despite the fact that the initial bulk modulus of $\mathrm{NSx} 30$ is lower than that of NSx5, for large compressive deformations $\left(\epsilon_{V}<-2<0 \%\right)$, NSx30 seems to be stiffer. Measurements conducted on pure silica for the compressive part [50,51] show similar shape and pressure values as the simulated curves.

From the polynomial fit of the hydrostatic deformation test, the apparent tangent bulk modulus is derived as $K \approx$ $-V_{0} d p / d V$ [53]. Figure 2 shows the tangent bulk modulus as a function of pressure for the different glass compositions. The $K-p$ curve is obtained from $p\left(\epsilon_{V}\right)$ and $K\left(\epsilon_{V}\right)$, and shows the tangent bulk modulus as a function of an easily measurable quantity. At zero pressure, the apparent bulk modulus of the different simulated glass compositions is consistent with experiments and previous calculations within $10 \%$ accuracy $[35,56]$ (see Table I).

In compression (positive pressure), NSx15 and NSx30 show a monotonous behavior from 0 to $20 \mathrm{GPa}$, where the tangent moduli evolve in the range of 45 to $110 \mathrm{GPa}$. For low sodium content (NSx5), however, a stiffness anomaly (nonmonotonous behavior) [57-59] is clearly observed. The tangent bulk modulus decreases until the minimum value 
TABLE I. Bulk modulus comparison between different calculation techniques and experiment [56]. Unit is in GPa.

\begin{tabular}{lccc}
\hline \hline Composition & This study & Ref. [35] & Experiments [56] \\
\hline NSx5 & 48.35 & $51.03 \pm 1.5$ & \\
NSx15 & 35.11 & $38.66 \pm 2.0$ & 36.03 \\
NSx30 & 36.07 & $37.92 \pm 1.8$ & 40.15 \\
\hline \hline
\end{tabular}

of 33.6 $\mathrm{GPa}$ (reached at $p=7 \mathrm{GPa}$ ), and then it increases again for higher pressure values. Similar results were already obtained in Ref. [56] where it was shown that $15 \% \mathrm{Na}_{2} \mathrm{O}$ was a critical composition separating anomalous behavior (for lower sodium content) to normal behavior. The anomalous nonmonotonous behavior may be related to the free open space in the structure and the role of sodium as lattice modifier. As shown in Fig. 3, when the pressure increases in low sodium content materials, structural changes affect mainly $\mathrm{Na}-\mathrm{Na}$ and $\mathrm{O}-\mathrm{O}$ interatomic distances [as shown in the partial structure factors, Figs. 3(b) and 3(c)], while the Si skeleton becomes slightly more disordered [Fig. 3(a)]. The interatomic $\mathrm{Na}-\mathrm{Na}$ distance changes by $33 \%$ for a global volume variation of only $10 \%$ when the pressure increases from 0 to $5 \mathrm{GPa}$ in NSx 5. As a result of these microbuckling events, the apparent tangent bulk modulus decreases [31,34]. In NSx30, the free volume is filled with sodium. The interactions of sodium ions with the Si skeleton are complex because sodium ions are more mobile, inducing local softening [35], but their presence prevents, in the same time, the isotropic collapse of Si rings. Therefore, initially softer, the material finally stiffens rapidly.

From several pressurized states, decompression was performed to relax the total pressure, and the resulting permanent relative volume change $\epsilon_{V}^{p l}$ was measured by comparing the initial and the final configuration in the same zero-pressure state. An example of a compression-decompression cycle is shown in Fig. 1 for NSx30. Figure 4 shows the permanent volume change which corresponds to the densification after the loading-unloading cycles until the maximum pressure value of $45 \mathrm{GPa}$. A sigmoidal fit [6] is used to guide the eye of the reader. All simulated compositions are shown next to two experimental measurements obtained for pure silica [54,55] and soda-lime silica [47]. At low pressure values, higher densification is observed in NSx30. But after reaching a limit pressure ( $5 \mathrm{GPa}$ in NSx5), the permanent volume change begins to increase rapidly, eventually saturating to a threshold $\left(\epsilon_{V}^{p l, \max }=-25 \%\right.$ in NSx5). This result is consistent with the phenomena observed in Fig. 2. A large increment of the permanent volume change shows an increase in volumetric plasticity, which may result in a decrease of the observed tangent bulk modulus with pressure as already discussed in [34]. The decay of the apparent modulus with pressure can also be related to any kind of irreversible rearrangement
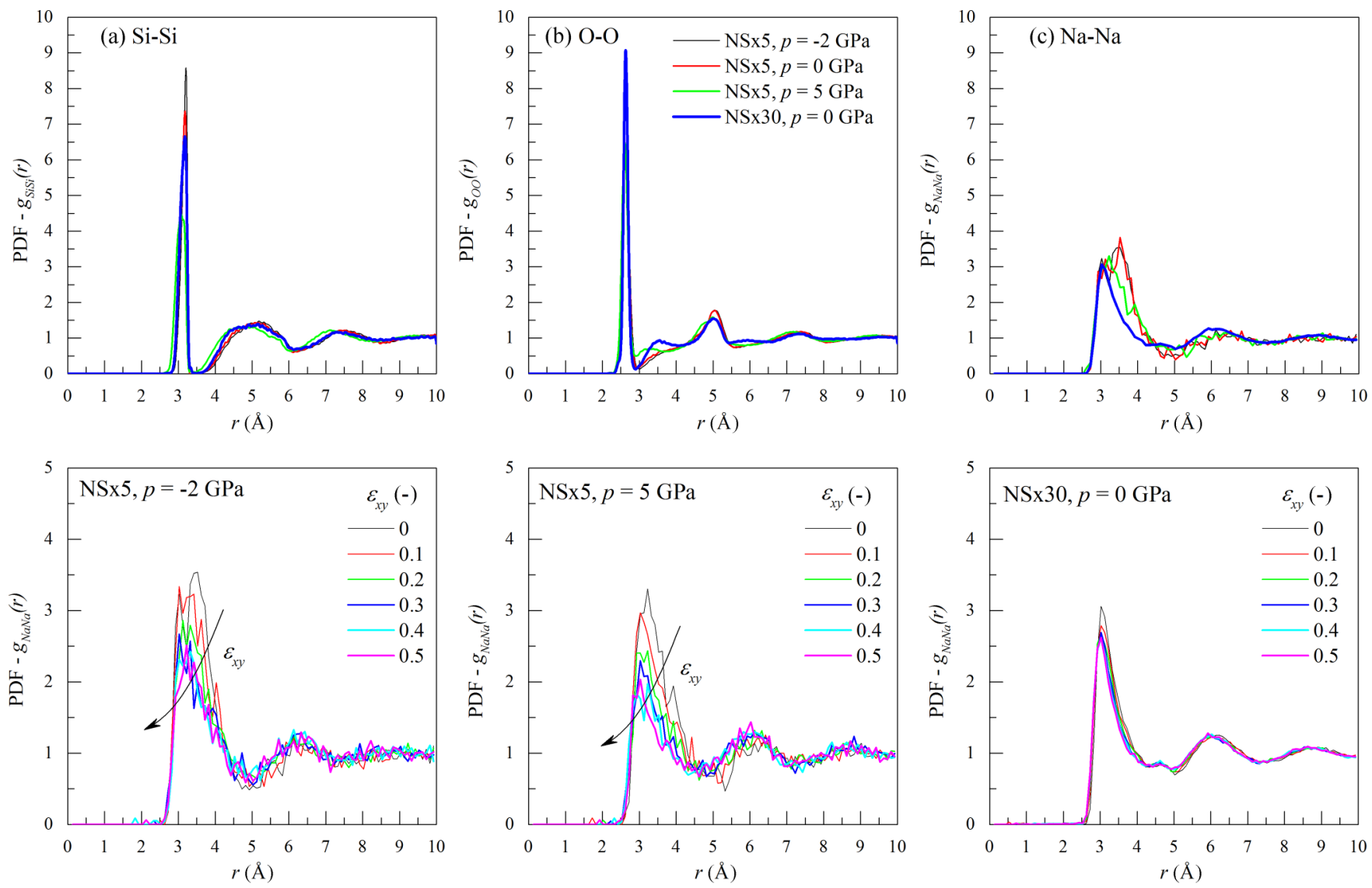

FIG. 3. Top: Partial pair correlation function for different compositions and different pressures. Bottom: Effect of shear strain on the Na-Na pair correlation function, for different compositions and different pressures. 


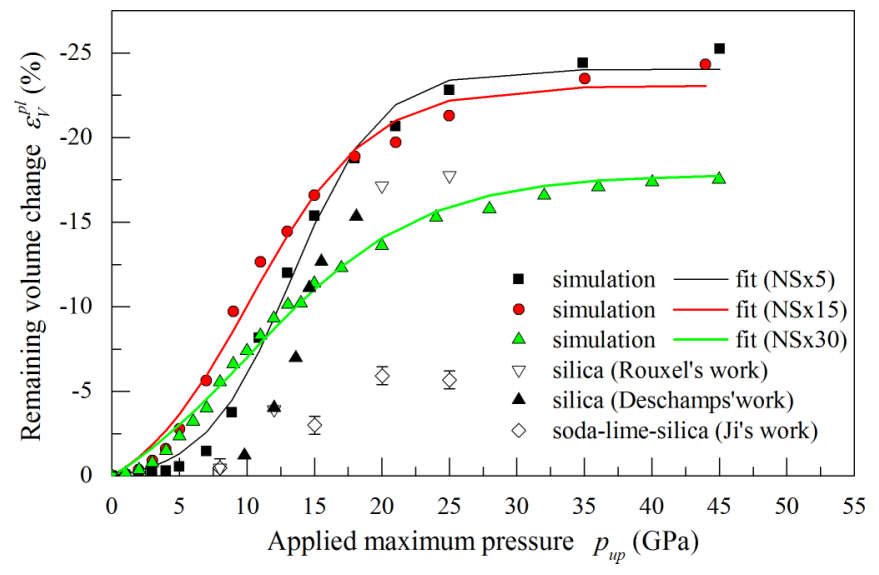

FIG. 4. Permanent volume change for NSx5, NSx15, and NSx30 glass samples obtained as a function of maximum pressure. The experimental result on pure silica [54,55] and a soda-lime-silica glass [6] is reported to compare the tendencies.

leading to a new atomic equilibrium position. In some cases, as will be shown later, these rearrangements can be driven by local irreversible shears only, without volumetric plasticity, especially at low pressures or for large sodium content. The idea that lower sodium content contains more free volume is confirmed here by the fact that the maximum volume change is significantly lower for $\operatorname{NSx} 30\left(\epsilon_{V}^{p l, m a x}=-17 \%\right.$ for NSx30).

Despite the fact that the present numerical models show higher permanent volume change than in experiments, the qualitative relation between the increase of the network modifiers concentration and the decrease of the permanent deformation is in good agreement with measurement [54]. Generally, the hydrostatic behavior of the simulated systems is in qualitative agreement with experiments [54,55] and density measurements [55,60], as seen in Figs. 1 and 4. This agreement strongly strengthens our numerical analysis.

\section{B. Shear deformation}

Quasistatic shear deformation is conducted in the $x y$ plane until $\epsilon_{x y}=50 \%$ tensorial shear strain for the different glass compositions and different pressure states (the prepressurized samples are taken from the previous part).

Figure 5 shows the composition dependence of the governing stress at $p=0 \mathrm{GPa}$ for sodium silicate glasses. The result for pure silica from the numerical work of Mantisi et al. [34] is also plotted in order to show the gradual change with the percentage of sodium content. As in pure silica, the shear response of low sodium content glass (NSx5) shows a softening stage which is characterized by a local maximum followed by a decrement. Finally, a plateau is reached for high strain values in all compositions. The increment of sodium content reduces the global shear stiffness, as can be observed by looking at the slope of the quasielastic part $\left(\epsilon_{x y}=[0,5] \%\right)$ that was drawn in Fig. 2. The material yields at a lower deformation stage and a lower yield strength, thus reducing as well the final yield strength at high strain values. Moreover, it appears that for a sufficient amount of sodium, the softening

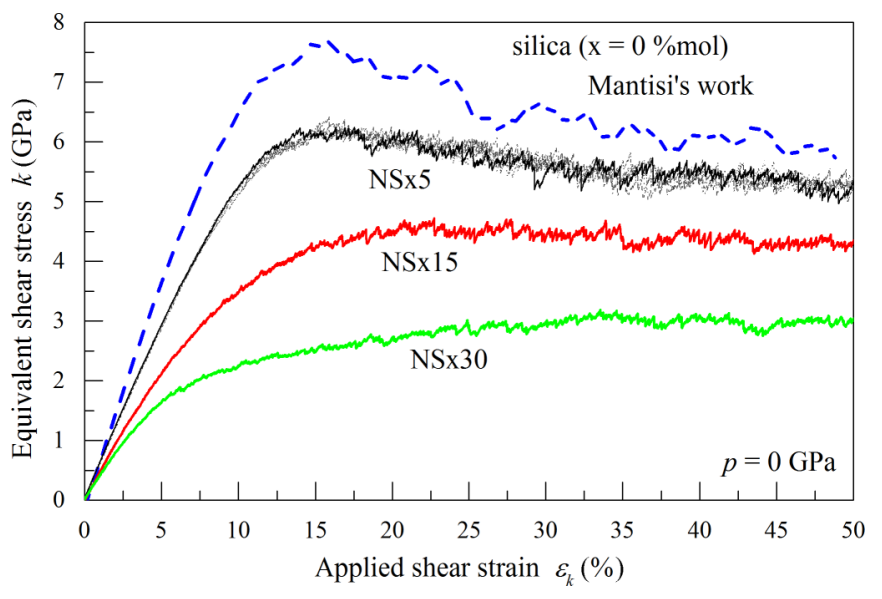

FIG. 5. Equivalent shear stress $(k)$ at $p=0 \mathrm{GPa}$ pressure as a function of the applied shear strain for different compositions, and four different configurations for NSx5. The result for silica is taken from the work of Mantisi et al. [34].

vanishes: for NSx30 and NSx15 glass, a continuous stress increase is observed without any significant decay.

Let us now consider the effect of pressure for a given composition. Figure 6 shows the equivalent shear stress $k$ as a function of shear strain for several constant pressure states ( $p=-10,-2,0,5 \mathrm{GPa}$ ) in NSx5. Using a dashed line (the same way as done in Fig. 1), the shear stress during unloading is shown in order to demonstrate the measurement of permanent plastic shear strains $\left(\epsilon_{x t}^{p l}\right)$. Two different responses can be distinguished in this figure:

Brittle. At high tensile stress (e.g., $p=-10 \mathrm{GPa}$ ), after a maximum shear stress the material completely loses its load bearing capacity and both shear stress and pressure reduce to zero. This large strain response is characterized by macroscopic crack formation.

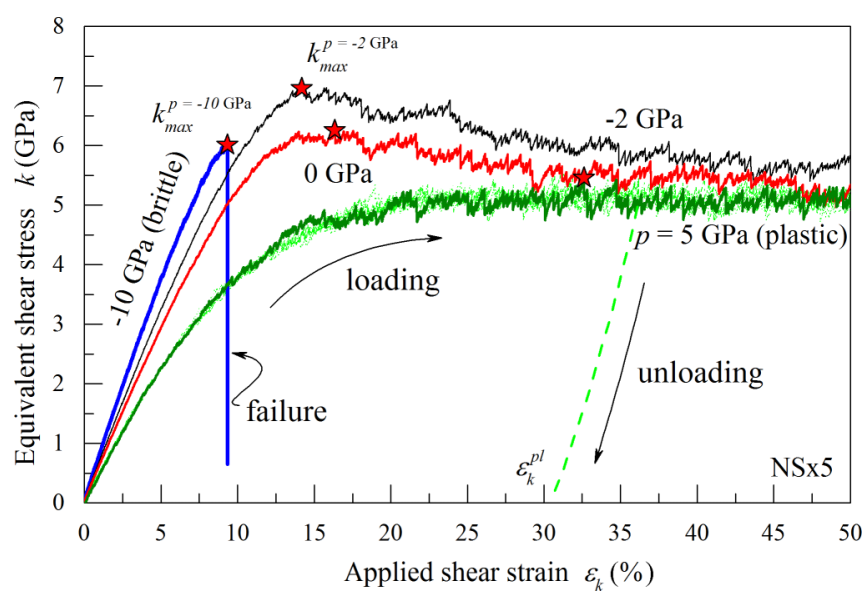

FIG. 6. Equivalent shear stress $(k)$ as a function of the applied shear strain for different pressure states and different configurations of NSx 5 glass. The figure shows the brittle failure at $p=-10 \mathrm{GPa}$ and the plastic response for the other cases. Red stars indicate the maximum shear strength achieved during deformation. Using a dashed line, a reverse loading case is shown to demonstrate the calculation of plastic shear strains $\left(\epsilon_{x y}^{p l}\right)$. 


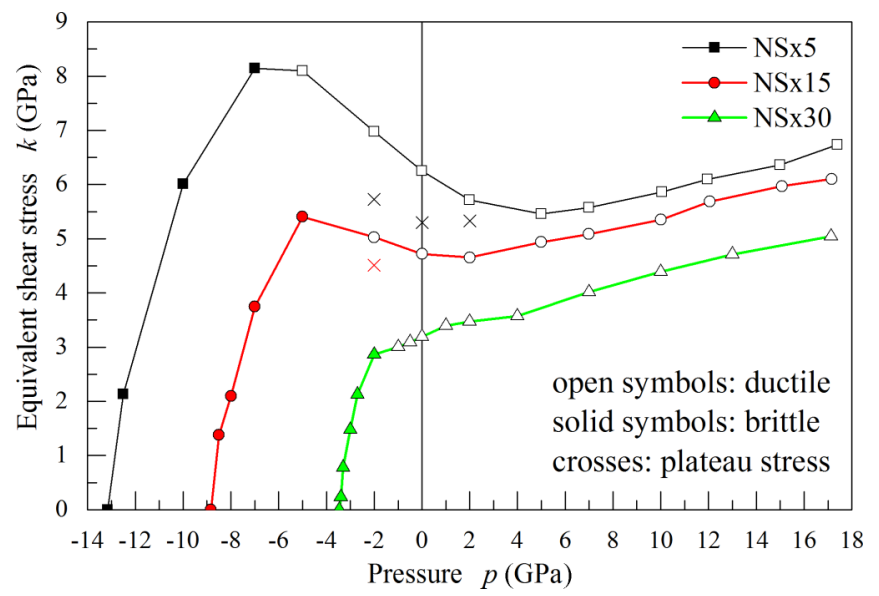

FIG. 7. Maximum shear stress (red stars in Fig. 6) as a function of pressure for NSx5, NSx15, and NSx30 samples. The solid symbols indicate that the failure is brittle. Plastic response is shown using open symbol. Crosses indicate the saturated value of the shear stress at large strains when it is different from the maximum shear stress.

Ductile. For higher pressure values, the applied shear strain can be increased without the disintegration of the sample. In this case, after either a softening stage (for low pressures) or a monotonic increment (for higher loads), the shear stress enters and maintains a plastic plateau. In this way, the tangent stiffness reduces to zero, but the pressure and shear stress state is maintained at a nonzero value.

In Fig. 7, the maximum shear stress is summarized as a function of the applied pressure for different compositions. Solid symbols show brittle failure modes and empty ones show the ductile failure modes. By adding $\mathrm{Na}_{2} \mathrm{O}$ into silica, the maximum shear stress and especially the tensile stress reduce significantly. The boundary between brittle and ductile failure, however, increases in the direction of the positive pressure. In NSx 5 , the first ductile pressure state is at $p \geqslant-5 \mathrm{GPa}$, for NSx15 it is $p \geqslant-2 \mathrm{GPa}$, and for NSx30 it is $p \geqslant-1 \mathrm{GPa}$. This means that not only the shear, but also the tensile strength of the material is reduced by the presence of sodium ions. A local maximum in the shear load bearing capacity is present for NSx5 and NSx15 as a function of pressure at $p=-6$ and $p=-5 \mathrm{GPa}$, respectively, after which the maximum shear stress reduces to a local minimum ( $p=5$ and $2 \mathrm{GPa}$ ) for NSx 5 and NSx 15 . These results show that pressure and, hence, permanent volume loss alter the structure causing the maximum shear stress to reduce. However, after this local minimum, the maximum shear stress increases again with the compaction of the material, as shown for other materials [61-63]. Indeed, it is interesting that permanent volume loss and pressurization do not affect NSx30 this way. The shear load bearing capacity of sodium rich samples increases monotonously with pressure.

Section IV will be dedicated to giving a microscopic explanation of the reduction of the supported stress with pressure and with the increasing amount of sodium.

\section{Remaining plastic deformation}

To map the plastic response of the material, the procedure described in Sec. II was systematically carried out. The samples were successively compressed isotropically to reach the target pressure, then sheared at constant pressure. After reaching the desired stress state $(p-k)$, the deformation was reversed and both shear stress and pressure were relaxed. Finally, the original and the final simulation box were compared to evaluate the remaining plastic strain tensor. Figure 8 shows two components of the plastic strain tensor as a function of the stress state $(p-k)$. Additional results can be found in Ref. [30] for different loading paths. Plastic shear strain $\left(\epsilon_{x y}^{p l}\right)$ is shown using red lines; permanent volume change $\left(\epsilon_{V}^{p l}\right)$ is indicated with black ones. To be able to compare the volumetric and shear strains, the volumetric strain $\left(\epsilon_{V}^{p l}\right)$ is divided by 3 and the tensorial shear strain $\left(\epsilon_{x y}\right)$ is multiplied by $2[64,65]$. The axes are set to the same range to highlight the compositional difference. The curves shown in Fig. 8 are thus a visualization of the elastic domain and the governing yield surfaces in the stress space $k-p$, for different values of the remaining plastic strain components. They allow us to compare the effect of stress and of sodium content directly on the plastic response, as already suggested in Ref. [34]. The pure elastic response can
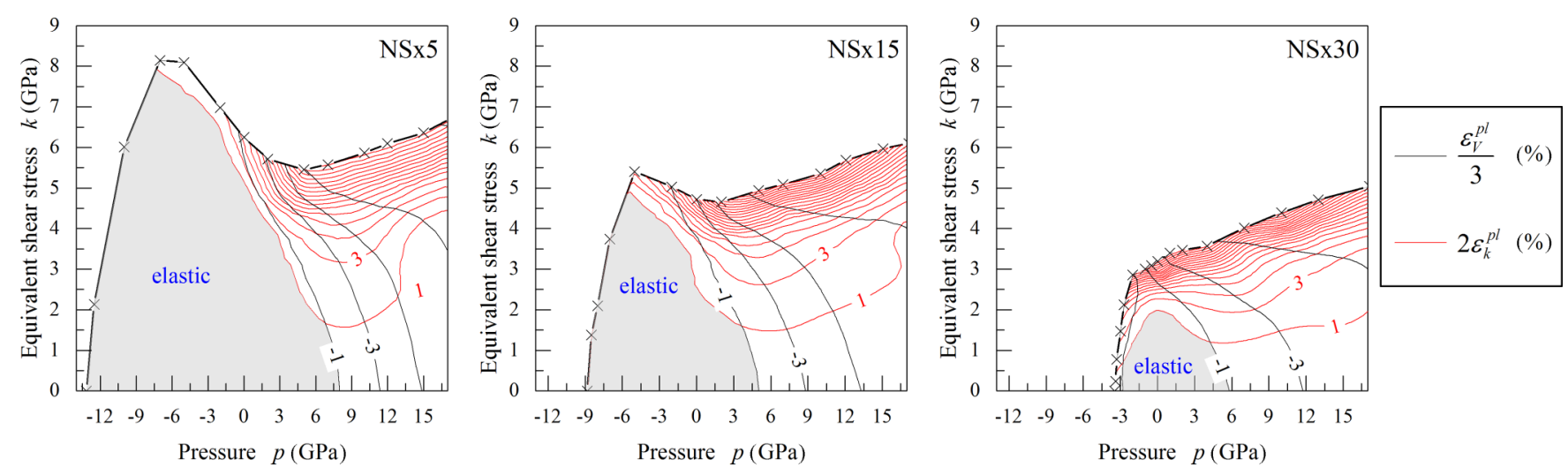

FIG. 8. Residual volumetric strain $\left(\epsilon_{V}^{p l}\right)$ and residual deviatoric (or equivalent shear) strain $\left(\epsilon_{k}^{p l}\right)$ as a function of maximum applied stress state for different compositions. Each numerical test is shown with crosses. Volumetric strain values are divided by 3 and deviatoric strain values are multiplied by 2 , as explained in the text. Every red curve symbolizes an increment in the permanent deviatoric deformation by $2 \%$, and every black curve does the same for the volumetric part. The gray area covers the quasielastic domain, where the plastic strains are lower than $1 \%$. 
only be found in the elementary deformation step; therefore, a quasielastic limit is defined at a threshold of the plastic strains equal to 0.01 (highlighted by the gray area). Using this representation, the role of sodium content on the elastic limit can be clearly identified.

Regarding the composition dependence, Fig. 8 clearly shows that the increase of the sodium content considerably reduces the elastic domain in the $k-p$ space. For NSx30, the elastic domain is strongly reduced, while NSx5 has a relatively large elastic domain where neither shear nor volumetric deformation is observed. Plasticity of NSx5 is mainly dominated by permanent volume loss which is different for NSx30, in which case the plastic shear strain is dominant in the early stages of plastic deformation, especially when low pressure is applied. Note that even for low sodium content, a small amount of plastic shear is always visible in the low pressure domain where no irreversible densification took place. However, the relative amount of volumetric and shear plastic strains depends on the composition: the plastic shear strain always increases with the amount of sodium.

Finally, it is interesting to recognize that volumetric plastic strain is increasing upon deviatoric deformation at constant pressure. For example, if a NSx5 sample is sheared at $p=$ $7 \mathrm{GPa}$ pressure, initially no permanent volume change is registered. If the shear stress exceeds $k=1.5 \mathrm{GPa}$, permanent volumetric strain increases up to $1 \%$. And, finally, at the maximum shear stress $k=5.5 \mathrm{GPa}, \epsilon_{V}^{p l}$ is more than $9 \%$. This coupling between applied shear and the resulting densification was observed experimentally a long time ago [66], but did not have clear evidence. The densification upon shear has a structural signature in the spatial distribution of sodium ions, which become closer to each other, especially at low pressures, as shown in Fig. 3. Based on our analysis, it was shown recently that shear and densification can indeed be taken into account in a single intrinsic parameter [30]. The overall decline of the load bearing capacity and the increase in the ductile behavior when increasing the sodium content is in agreement with experimental measurements [67].

We will now compare these global results to the analysis of the microscopic irreversible processes responsible for plastic deformation at small scales.

\section{LOCAL MICROSCOPIC ANALYSIS}

\section{A. Nonaffine displacements}

In previous sections, it was shown that the yield behavior of sodium silicates strongly depends on composition and pressure: Fig. 7 showed that the presence of sodium and the increasing pressure (and densification) reduces the shear strength significantly. To complete the previous global plastic description, a local analysis is now performed. To compare the effect of composition and pressure, four shear cases were chosen: NSx5 and NSx30 at $p=0 \mathrm{GPa}$ highlights the differences caused by sodium; and NSx5 at $p=-2$ and $5 \mathrm{GPa}$ shows the effect of pressure.

To identify the plastic activity, the nonaffine displacement field is used $[68,69]$. This quantity was shown to highlight the plastic centers that are located precisely at its local maxima

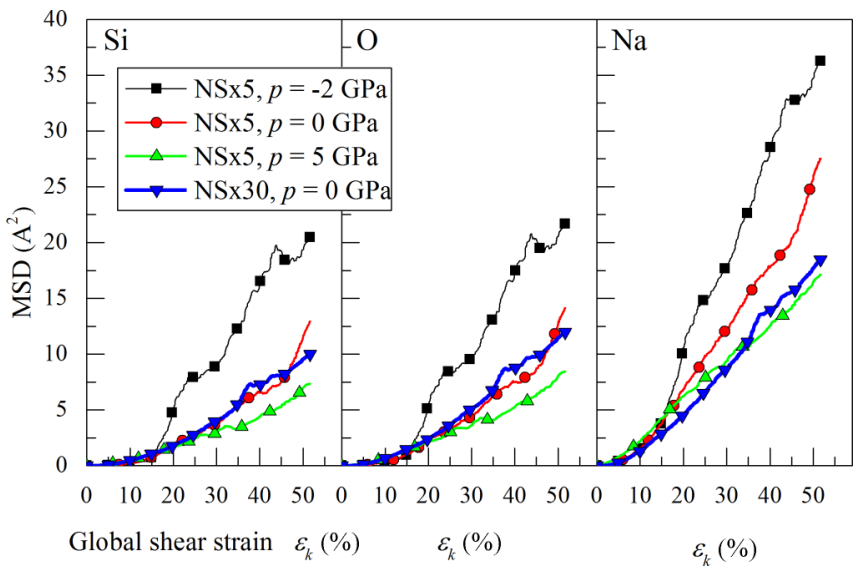

FIG. 9. Mean squared nonaffine displacement of $\mathrm{Si}, \mathrm{O}$, and $\mathrm{Na}$ atoms during shear deformation for NSx 5 at $p=-2,0,5 \mathrm{GPa}$ and NSx30 at $p=0 \mathrm{GPa}$ pressure.

as soon as irreversible displacement occurs [15,23,26,70]. The nonaffine displacement field $d \underline{u}_{n a}$ is obtained between two deformation states by subtracting the displacements of the atoms in the affine hypothesis to the real displacements obtained after mechanical equilibrium is reached. It is written as

$$
d \underline{u}_{n a}=d \underline{u}-d \underline{\varepsilon} \cdot \underline{r}_{0},
$$

where $d \underline{u}$ is the atomic displacement obtained from simulations, which compares the atomic positions after energy relaxation to the atomic positions before the external deformation is applied, and $d \underline{\varepsilon} \cdot \underline{r}_{0}$ is the so-called affine part where $\underline{r}_{0}$ corresponds to the initial position of the atom and $d \underline{\varepsilon}$ is the global strain step tensor between the deformation states. The nonaffine displacement field thus evaluates the atomic displacements occurring during energy minimization. It corresponds as well to the deviation from the homogeneous strain field. Its average over all the atoms is zero.

To accurately measure the displacements, $d \underline{u}_{n a}$ is calculated between every 10 load steps (e.g., between the initial and the configuration obtained after 10 load steps, then between the 10 th and 20th one, and so on) that is for a strain interval $d \epsilon_{x y}=10 \cdot(\delta \epsilon)=5 \times 10^{-2} \%$. The cumulative nonaffine displacements $\underline{u}_{n a}\left(\epsilon_{x y}\right)$ are obtained, if needed, by summing the nonaffine displacement increments up to the global strain $\epsilon_{x y}$ :

$$
\underline{u}_{n a}\left(\varepsilon_{x y}\right)=\sum_{d \varepsilon_{x y}} d \underline{u}_{n a} .
$$

Similarly to thermal diffusion in atomic systems, nonaffine displacement provides information about the random motion caused by shear, in the harmonic as well as in the anharmonic regime. In Fig. 9, the mean squared displacement $\left(\mathrm{MSD}_{n a}\right)$ is shown as a function of the applied shear strain. The displacement data is shown for the four chosen deformation cases and each atomic species is plotted in a separate figure. $\mathrm{MSD}_{n a}$ is calculated by averaging the cumulative nonaffine displacement over different atomic species:

$$
\operatorname{MSD}_{n a}\left(\varepsilon_{x y}\right)=\frac{1}{N} \sum_{i=1}^{N}\left[u_{n a}^{i}\left(\varepsilon_{x y}\right)\right]^{2}
$$


where $N$ is the number of atoms in each species and $u_{n a}^{i}\left(\varepsilon_{x y}\right)$ is the amplitude of the cumulative nonaffine displacement vector. This quantity shows the relative mobility of the atoms. During shear deformation, the network modifier ( $\mathrm{Na}$ atoms) appears to be the most mobile atomic species, followed by oxygen and silicon atoms. This order respects as well the mobility observed by thermal activation [71-73]. It appears that reducing the free volume in the structure (by compressing the samples) reduces the global mobility of the atoms. Sodium atoms motion also decreases by increasing the composition (in this way, filling up the free volume with sodium) from NSx 5 to NSx30. However, the motion of the other two species ( $\mathrm{Si}$ and $\mathrm{O}$ ) is not affected significantly by the amount of network modifiers. These atomic scale measurements are in agreement with the interpretation of the global elastoplastic response proposed in Sec. III A in terms of atomic mobility and free volume in the silicon skeleton.

The nonaffine displacements can also be used to identify local plastic events (PEs), which control the major part of irreversible rearrangements in amorphous solids [12,15,74]. Fusco et al. [20,23] showed that the dissipation of elastic energy is mainly located at the position of the local maxima of the nonaffine displacement fields. We will now look in detail at the core of the plastic events.

\section{B. Plastic events}

From the atomic displacements, it is possible to compute local strains by using, for example, the physically based coarse-graining procedure proposed by Goldenberg et al. [70]. The definition of strain is then obtained by deriving the coarse-grained continuous displacement field, whose expression preserves the mass conservation equation. We applied this coarse-graining procedure to the nonaffine displacement values to transform the original discrete atomic values into a continuum field. More precisely, the technique consists of a mass-weighted Gaussian convolution evaluated on a 3D grid with $100 \times 100 \times 100$ points. A coarse-graining length, which is basically the width of the Gaussian function, is set to $3 \AA$ according to the work of Goldenberg et al. [70]. With this method, the discrete values calculated at each atom are weighted differently to obtain a homogeneous field. Using the definition of the Green-Lagrange strains (e.g., $2 \varepsilon_{x y}=$ $\frac{\partial u}{\partial y}+\frac{\partial v}{\partial x}+\frac{\partial u}{\partial x} \frac{\partial u}{\partial y}+\frac{\partial v}{\partial x} \frac{\partial v}{\partial y}+\frac{\partial w}{\partial x} \frac{\partial w}{\partial y}$, where $u, v$, and $w$ are displacements in the $x, y$, and $z$ directions, respectively), $\epsilon_{x y}$ can be calculated locally from the coarse-grained displacement field. To obtain this last quantity, a larger $5 \AA$ coarse-graining length was used because it was shown previously [35] that strain is ill defined under this value.

Figure 10 compares the amplitude of the coarse-grained cumulative nonaffine displacement field $u_{n a}(x, y, z)$, and of the plastic shear strain field $\epsilon_{x y}^{p l}(x, y, z)$ for different cases in the middle plane of the sample. The plastic shear strain $\epsilon_{x y}^{p l}(x, y, z)$ was calculated by comparing the initial (undeformed) sample and the one which was relaxed backward from $\epsilon_{x y}=20 \%$ to $\sigma_{x y}=0$ (in both cases, the global shear stress is zero). The comparison between the plastic strain field and the nonaffine displacement, cumulated up to $\epsilon_{x y}=20 \%$, definitely strengthens in our specific sample the relationship between
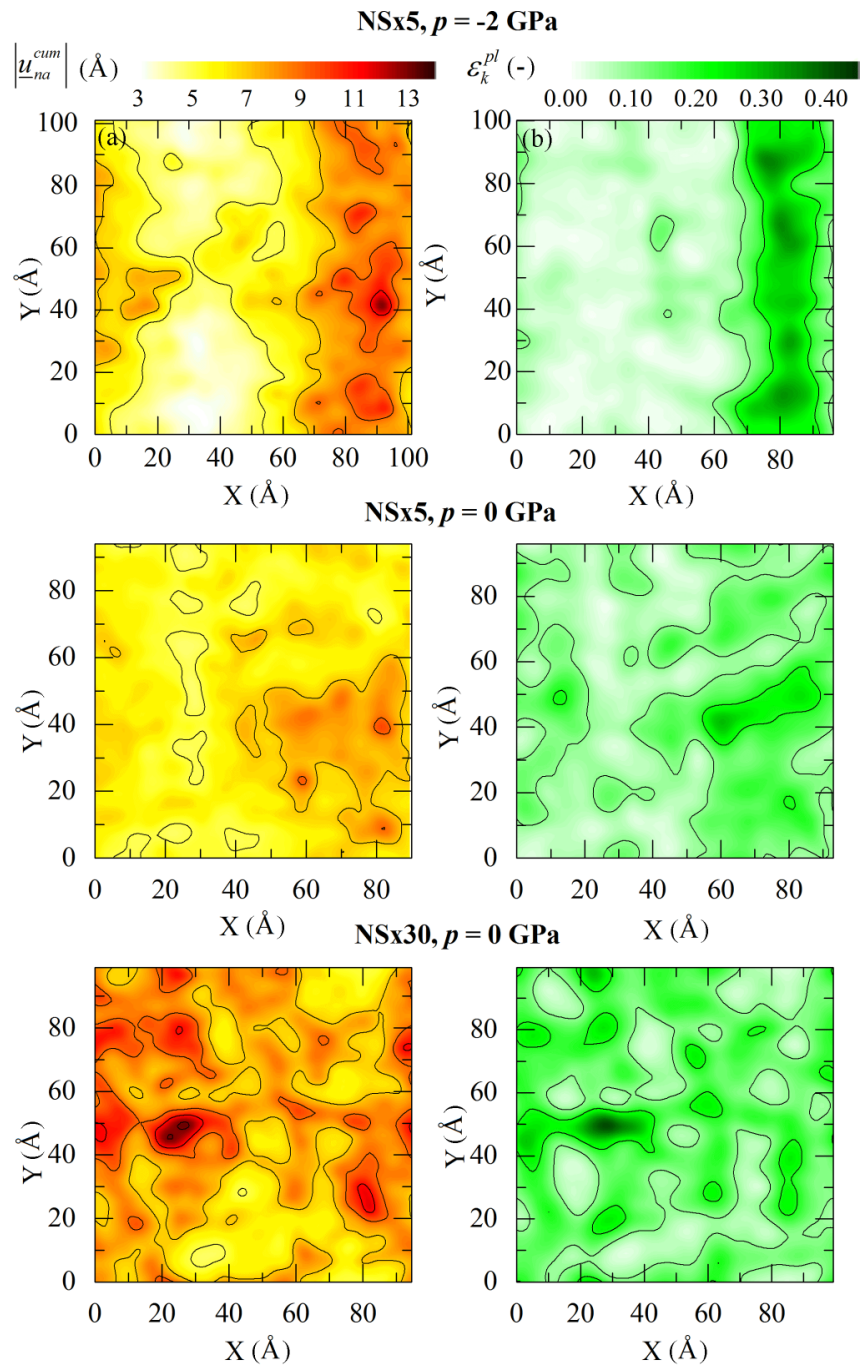

FIG. 10. Amplitude of the cumulative nonaffine displacement field at $\epsilon_{x y}=20 \%$ (left) and permanent plastic shear strain $\left(\epsilon_{x y}^{p l}\right)$ after relaxing shear stresses (right) from the same deformation state. The results are shown for NSx 5 at -2 and $0 \mathrm{GPa}$, and NSx30 at $0 \mathrm{GPa}$. The correlation coefficient computed for the data sets is $\operatorname{corr}\left(\left|\underline{u}_{n a}^{\text {cum }}\right|, \epsilon_{x y}^{p l}\right)=0.83,0.72,0.78$, respectively.

nonaffine displacements and globally calculated plastic strains: Strong spatial correlations $\left(\operatorname{corr}\left(\left|u_{n a}\right|, \epsilon_{x y}^{p l}\right)=0.83,0.72,0.78\right)$ indeed show that the cumulative nonaffine displacement field is directly related to the governing plastic strain. This measurement confirmed that plastic rearrangements identified by the nonaffine displacements are truly responsible for the major part of global plastic strain, not just statistically but spatially as well. In NSx5 samples at $p=-2 \mathrm{GPa}$, the localization of both the nonaffine and the plastic strain field along elementary shear bands can be observed. Note that in this deformation case, at $\epsilon_{x y}=20 \%$, the stress-strain relationship shows a softening response (Fig. 6). This result supports the theory that shear softening is related to the persistent alignment of plastic events (PEs) [27,75,76]. By looking at the other two cases in Fig. 10, it is seen that the increment of either pressure or sodium content homogenizes the plastic response. At the same time, no shear softening was observed: the samples harden monotonously. 


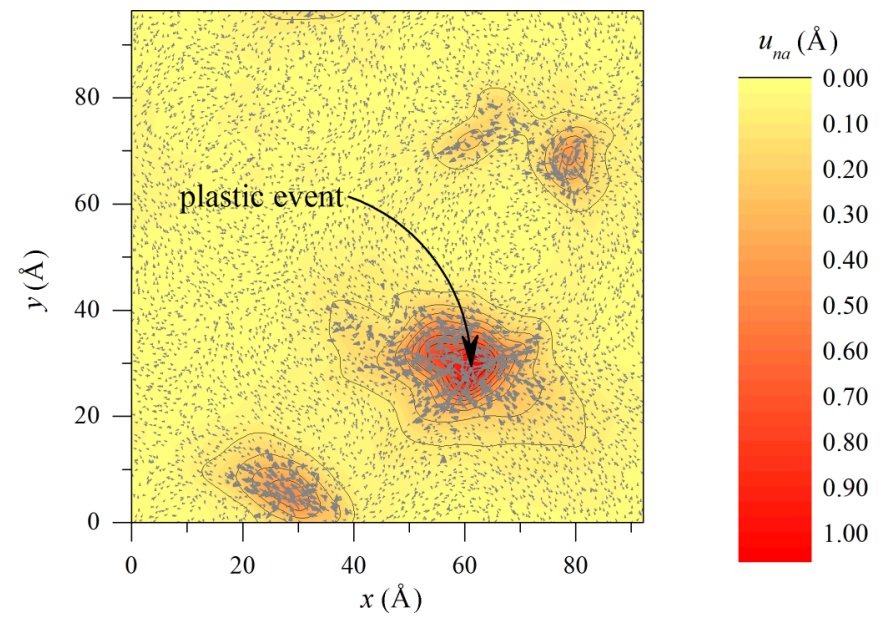

FIG. 11. Amplitude of the coarse-grained nonaffine displacement field in NSx5 sample at the plastic event (PE). The figure shows the position of the plastic event with a curved black arrow, and the planar projections of atomic displacements are drawn with gray arrows.

The contribution of the individual rearrangements to the global plastic strain can be studied in detail as a function of the composition and pressure. The characteristics of the PEs are obtained using the method developed by Fusco et al. [20]. First, $d u_{n a}(x, y, z)$ was computed between every 10 load steps. Then the local maxima of this field are identified as individual PEs. According to the recommendation of Ref. [20], local maxima smaller than $d u_{n a}<0.1 \AA$ are neglected because their amplitude is within the numerical precision of the energy minimization scheme. Figure 11 shows the amplitude of the coarse-grained nonaffine displacement field of the NSx5 sample with $p=0 \mathrm{GPa}$ in the middle plane $(z=50 \AA)$ for $\epsilon_{x y} \in[15.50,15.55] \%$ (10 load steps). A PE is clearly shown at $x=62 \AA$ and $y=29 \AA$. It corresponds to a local plastic strain with evident contribution of shear displacements, analogous to the Eshelby-like strain heterogeneities recently recognized as the elementary ingredient for plastic deformation in amorphous materials $[17,19]$. Plastic strain is given directly by the eigenstrain of the Eshelby inclusions. The size $\left(r_{P E}\right)$ of a PE is estimated by looking at the radial decay of the angular averaged intensity of the nonaffine field around its local maximum. The values were then fitted with an exponential function: $u_{n a}=u_{n a}^{\max } \exp \left(-r / r_{\mathrm{PE}}\right)$, where $u_{n a}^{\max }$ is the value at the peak, $r$ is the radial distance, and $r_{P E}$ measures the radius of the PE's core. It is possible to measure not only the size and the number of plastic events, but also their core's composition, and internal strain tensor. The results are summarized in Fig. 14.

Figure 14(a) shows the cumulative number of PEs calculated by counting the local maxima at every 10 step interval. Similarly to the evolution measured in other systems such as amorphous silicon [20], three stages are clearly visible: (1) a first stage where almost no PE is found, (2) a second stage where the PEs appear, and (3) a final stationary stage, where the number of PEs appearing at each strain step is constant, giving rise to a linear strain dependence of the cumulative number of PEs, or constant nucleation rate. The first stage can be considered as elastic (plasticity is negligible); then the material gradually plastifies, and finally the response enters a stationary plastic plateau. The second stage appears as a crossover between the elastic regime and the perfect plastic plateau. The crossover between these two regimes can be fitted assuming a hyperbolic transition [fits shown in Fig. 14(a)]. It shows a characteristic crossover strain $\epsilon_{k}^{C}$ depending on the external pressure and on the glass composition, while the final creation rate depends only on the composition. For NSx 5 at $p=0$ and $-2 \mathrm{GPa}$, the elastic regime is found until $\epsilon_{x y}=5 \%$; in contrast, $\mathrm{NSx} 30$ at $p=0 \mathrm{GPa}$ and NSx5 at $p=5 \mathrm{GPa}$ begin to yield at an earlier strain state $\left(\epsilon_{x y}=2-3 \%\right)$. The resulting number of plastic events is always larger for large sodium content (NSx30) or high pressures (NSx5 at $5 \mathrm{GPa}$ ). It is also shown that in the final stationary regime, all the samples containing $5 \% \mathrm{Na}_{2} \mathrm{O}$ have similar plastic rate: the differences in the plastic rates, due for example to pressure variations, are restricted to the initial stages. In contrast, in $\mathrm{NSx} 30$ the plastic rate in the stationary regime is almost two times larger compared to NSx5. The role of composition is thus crucial for the plastic activity, but interestingly the number of plastic events alone is not sufficient to reconstruct the global stress-strain curves.

Figure 14(b) shows the amplitude of the nonaffine displacement field at the plastic event averaged over all the events in the same strain step. The same kind of stages can be found as in Fig. 14(a): after an initial low value, the amplitude increases quasilinearly and saturates to a stationary regime. It can be observed that the amplitude in NSx30 is generally lower in all stages; however, it finally saturates to a value similar to the pressurized NSx5 sample. Not just composition but also pressure have a significant effect on the amplitude: increasing the pressure enhances the amplitude in the initial quasielastic stage, but the amplitude decreases with pressure in the final stationary plateau. The general trend is thus an enhancement of plastic activity with pressure, combined with slightly larger amplitude events in the early stages of deformation, and then significantly lower amplitude. Increasing the amount of sodium content always increases the plastic rate but decreases the amplitude of plastic rearrangements. We will now look at the effective volume involved in the plastic rearrangements.

Figure 14(c) shows the average radius size $\left(r_{\mathrm{PE}}\right)$ of the plastic event's core as a function of the imposed shear deformation. In all cases, the PE's radius is smaller in the early stage, but increases gradually and tends to a stationary value in the plastic plateau. Initially, $r_{\mathrm{PE}}$ is clearly pressure and composition dependent. It is enhanced by the pressure and by the sodium content. Increasing the pressure from -2 to $5 \mathrm{GPa}$ in NSx 5 samples enlarges the plastic cores by $50 \%$ from 4 to $6 \AA$. This effect is similar to the composition effect: higher sodium content increases the core's size in the same range. In the stationary regime, the PE core radius is nearly $6.5 \AA$ and it is only slightly enlarged by pressure but no longer depends on composition.

The distribution of the different strain components measured in the core of the inclusions at each deformation step and weighted by the size of the PEs is shown in Fig. 12. In agreement with the previous analysis, two regimes of deformation were distinguished: Figures 12(a) and 12(b) show the histograms of the different components of the strain tensor measured in the center of the inclusions for states belonging to the initial linear part of the global stress-strain relation. 


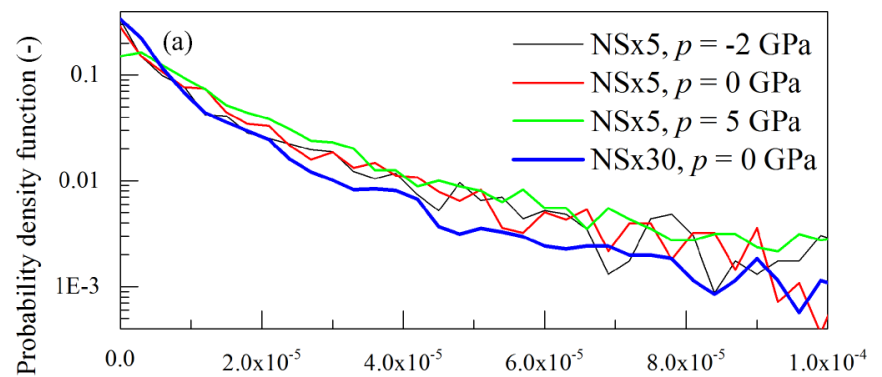

Local deviatoric strain weighted with the relative size of the PE (-)

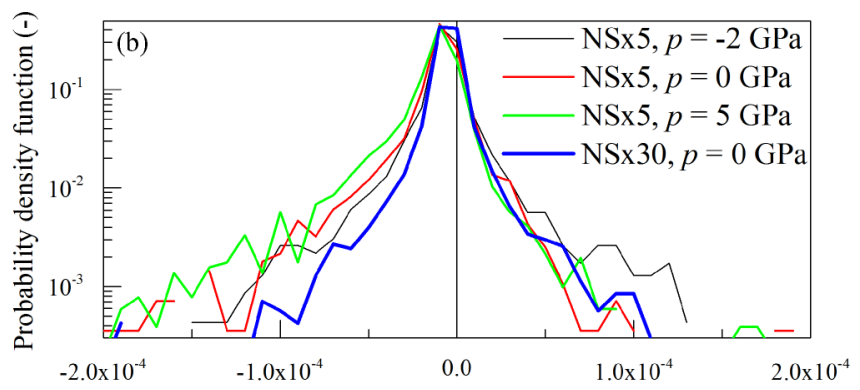

Local volumetric strain weighted with the relative size of the PE (-)

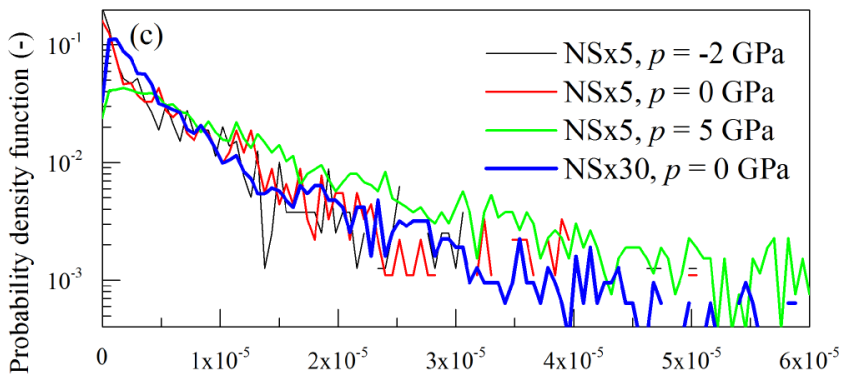

Local deviatoric strain weighted with the relative size of the PE (-)

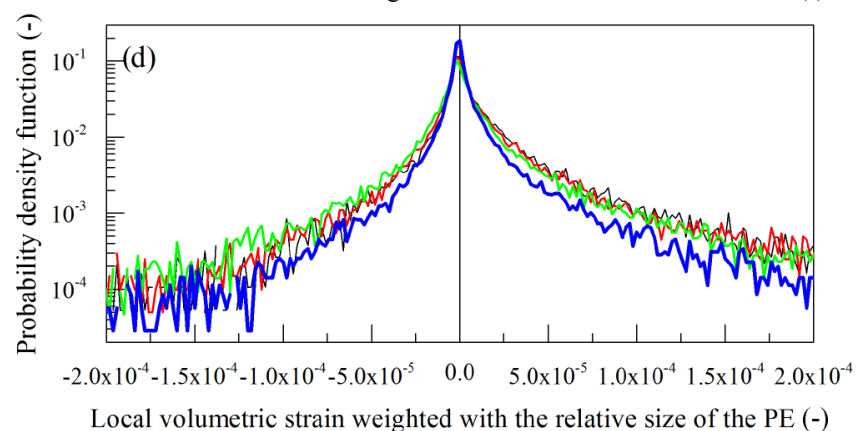

FIG. 12. Top: Distribution of (a) deviatoric strain components and (b) volumetric strain measured in the core of the plastic events in the linear part of the stress-strain relation. Bottom: Distribution of (c) deviatoric strain components and (d) volumetric strain measured in the core of the plastic event, in the plastic plateau. The strain values are computed in the center of the plastic events and are weighted by the volume of the plastic events supposed to be spherical, with the radius of the PEs core.

Figures 12(c) and 12(d) show the same histograms obtained in the plastic plateau. The histograms in Figs. 12(c) and 12(d) are better defined due to the larger number of plastic events in this part. Contrary to Ref. [19], here we show the histogram of the total strain, and not of the eigenstrain of corresponding Eshelby inclusions, because we have chosen to show the
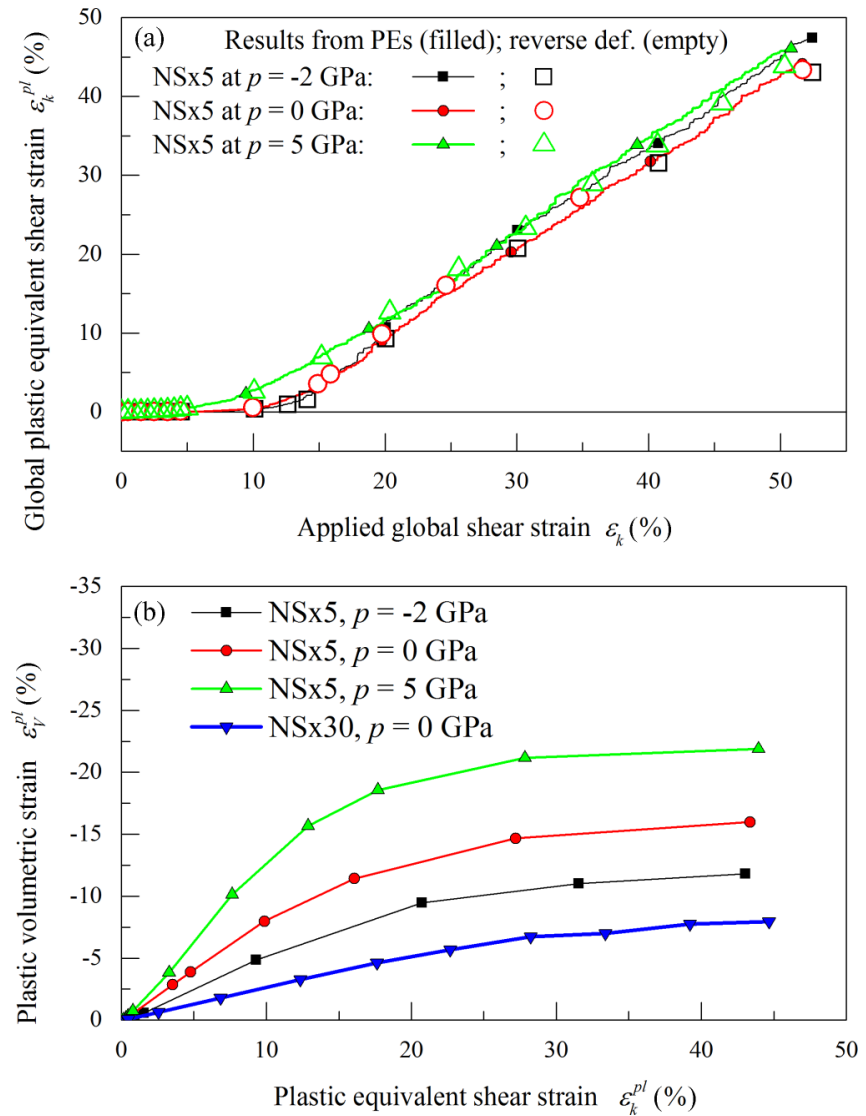

FIG. 13. (a) Global plastic equivalent shear strain $k_{\epsilon}^{p l}$ as a function of the applied global shear strain, for different compositions and pressures. The plastic equivalent shear strain is computed either from the reverse shear deformation (empty symbols) or from the deviatoric strain inside the PEs (filled symbols). (b) Plastic volumetric strain as a function of plastic equivalent shear strain for different compositions and pressures.

direct measurement of total strain without any assumption on the elastic strain. The result should be comparable to the distribution of true eigenstrains, as it was shown in Fig. 13(a), that the elastic contribution to the strain is negligible in the center of the PEs. Indeed, the total strain, computed as the sum of the local deviatoric strain measured in the centers of the PEs, compares quite well with the global plastic deformation measured after unloading the system. In particular, the slight increase of the remaining plastic shear strain when pressure increases is clearly visible in the figure for a global applied strain of $\approx 10 \%$. In Fig. 12, the strains are weighted by the relative size of the PEs, as suggested in Ref. [19]. We used the same quantity to compute the total averaged remaining plastic deformation in Fig. 13(a) mentioned above (results from PEs), assuming that the plastic deformation is localized strictly in the core of the PEs.

The histograms of the deformation in the core of the PEs show, in Fig. 12, that the distributions are not very different for different compositions and pressures. The main difference occurs in the volumetric part. There is a small, but not negligible, volumetric strain for all the events in the sample, with a larger distribution for compression than for expansion in the early stages of deformation [Fig. 12(a)]. It is 

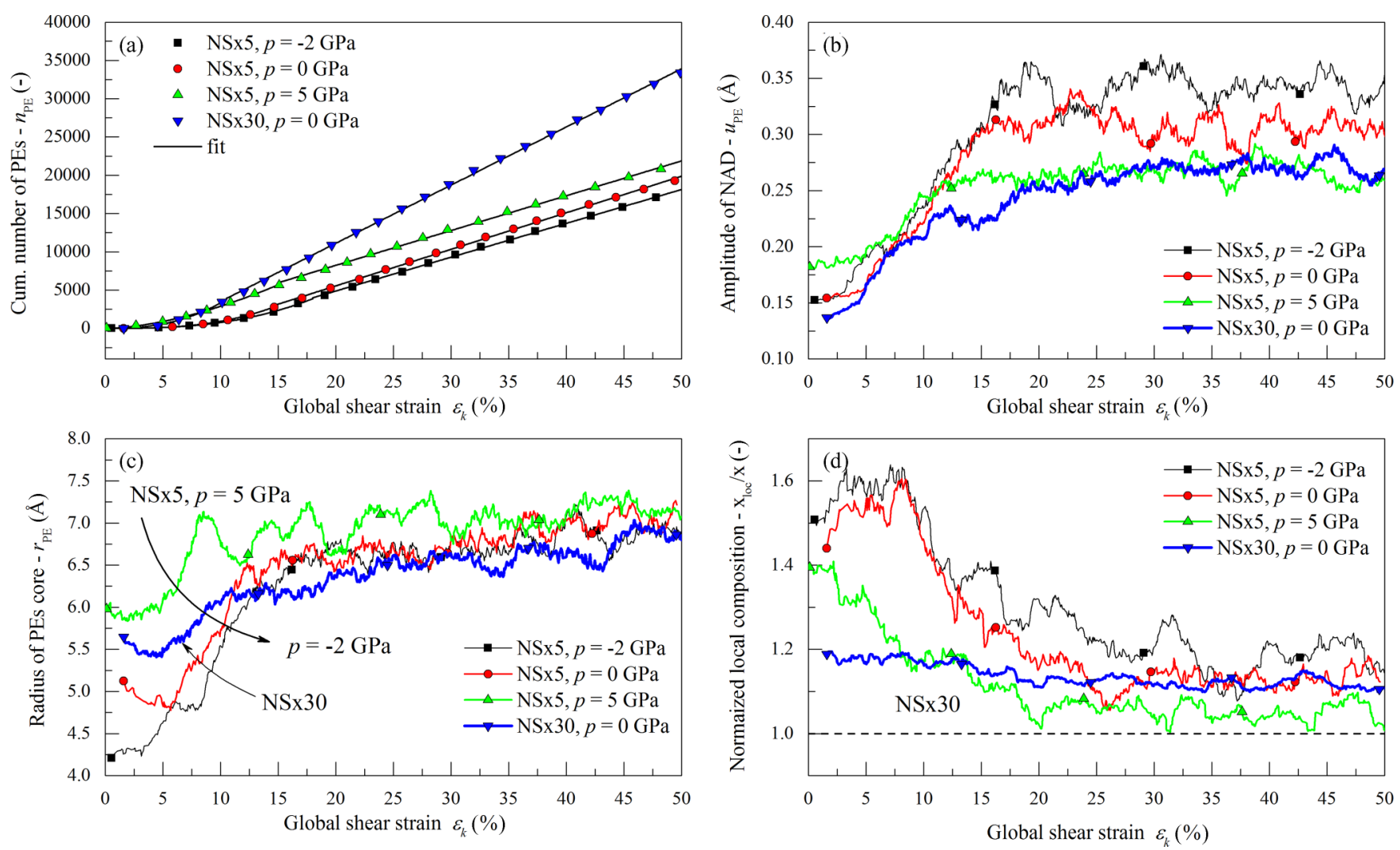

FIG. 14. (a) Cumulated number of plastic events during shear deformation for NSx5 at $p=-2,0,5 \mathrm{GPa}$ and NSx30 at $p=0 \mathrm{GPa}$. (b) Average amplitude of the nonaffine displacement field at the plastic event. (c) Average radius size $\left(r_{\mathrm{PE}}\right)$ of the core of plastic events as a function of applies global shear strain. (d) Average normalized local composition $\left(x_{\mathrm{loc}} / x\right)$ at the core of plastic events.

interesting to note that for low sodium content, larger pressure ( $p=5 \mathrm{GPa}$ ) will change the local volumetric strain, giving rise to larger local densification during applied shear. In the case of large sodium content (NSx30) in contrast, the local volume expansion is as important as the local compression, as in the case of low pressure low sodium content, but the local deviatoric strain is smaller. In general, large sodium content gives rise to smaller core strains. The total remaining plastic deformation results from the accumulation of PEs, and the large plastic capacity of NSx30 results thus solely from the larger number of (smaller size) events. In this case, the (non-negligible) densification is compensated by the (non-negligible) volume expansion, without significant global remaining volumetric strain. In all cases, the local deviatoric strains are larger or equal to the volumetric strains, and follow an approximately exponential distribution with characteristic value for the weighted deviatoric strain, $\left(\epsilon^{d}\right)=3 \times 10^{-3} \%$ $\left(L^{3} \cdot \epsilon^{d}=30 \AA^{3}\right.$, comparable to the value obtained in amorphous silicon [19]). This characteristic weighted strain is consistent with an averaged eigenstrain in the core of Eshelby inclusions, $\left(\epsilon^{*}\right) \approx 2 \times 10^{-3}$, with an average size $r_{\mathrm{PE}} \approx 6 \AA$ [19]. The local plastic strain is small, but the large number of plastic events explains the non-negligible remaining plastic strain values.

In the plastic plateau, the distribution of the volumetric changes is extended to larger volume variations. Upon shear at constant pressure, the densification reached can be larger than upon direct hydrostatic compression, as was already observed in Ref. [30]. Finally, in the plastic plateau, the plastic strain can reach a few tens of percents. The relation between the volumetric remaining plastic strain and deviatoric remaining plastic strain is shown in Fig. 13(b). In the plastic plateau, it becomes proportional, with slightly larger deviatoric strains. This figure also illustrates also the fact that the part of volumetric plastic strain increases with pressure, while deviatoric strain is dominant in samples with large sodium content. Despite the similar decrease of plastic yield in both cases, large pressure and large sodium content differ from the relative amount of volumetric versus deviatoric plastic strain.

Finally, the local composition in the core of the plastic events is calculated to identify to role of sodium. All atoms are counted inside the core of the plastic event $\left(r<r_{\mathrm{PE}}\right)$ and, using the following formula, the local composition $\left(\mathrm{x}_{\mathrm{loc}}\right)$ is obtained:

$$
\mathrm{x}_{\mathrm{loc}}=\frac{n_{\mathrm{Na}}}{n_{\mathrm{Si}}+n_{O}+n_{\mathrm{Na}}} \frac{3}{2},
$$

where $n_{\mathrm{Si}}, n_{O}$, and $n_{\mathrm{Na}}$ are the number of silicon, oxygen, and sodium atoms counted. In Fig. 14(d), the average local composition divided by the global value is shown as a function of applied strain for the four cases. The data are normalized by the average concentration of sodium in order to make the comparison easier. Here again, two regimes have to be distinguished. In the initial stages of plasticity, plastic events appear clearly at sodium rich regions. In this stage, for NSx5 at $p=-2$ and $0 \mathrm{GPa}$, the local composition of the PEs is 


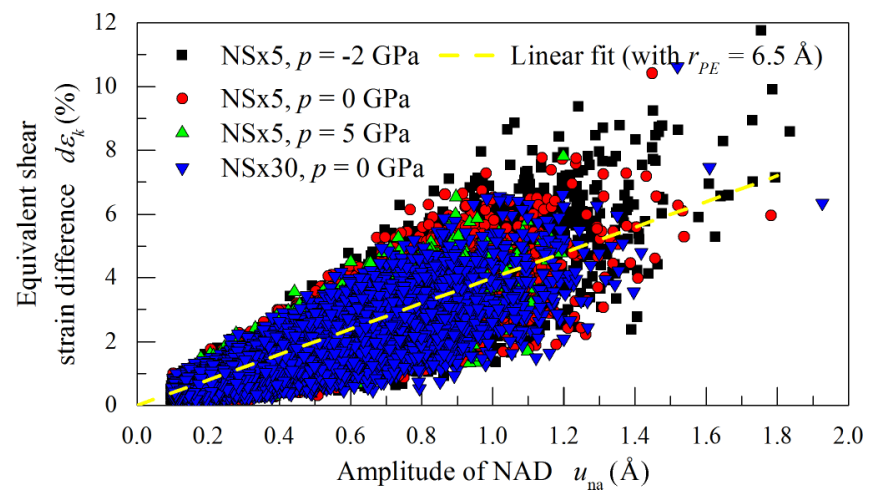

FIG. 15. Local shear-strain increment caused by PEs as a function of the amplitude of the nonaffine displacement registered at their core for NSx5 at $p=-2,0,5 \mathrm{GPa}$ and NSx30 at $p=0 \mathrm{GPa}$ with a linear fit [see Eq. (6)] corresponding to $r_{\mathrm{PE}}=6.5 \AA$.

$60 \%$ higher compared to the global value. For NSx30, the initial value is not as dominant $(\approx+20 \%$ compared to the average composition). The plastic activity near mobile sodium decreases progressively. But in any case, even after the plastic plateau is reached, the average sodium content in the core of the plastic events remains higher than the average concentration. This result clearly indicates that sodium atoms act as a catalyst for the early stages of the plastic deformation.

To summarize this analysis, we showed that it is necessary to distinguish three phases for the plastic behavior of silicate glasses: an initial stage with very low density of PEs, an intermediate regime that is crucial in determining the properties of the elastic limit, and a final stationary stage with a constant nucleation rate of events corresponding to a perfect plastic plateau in the global stress-strain behavior. The decay of the initial yield stress in the case of large sodium content samples is shown to be related to the large number of PEs in this case that are located close to the more mobile sodium ions. In the early stages of plasticity, the size of the events is larger for large sodium content samples, but the average weighted strain in the core of the plastic events is smaller. The larger nucleation rate of plastic events is thus crucial to explain the smaller yield stress in this case. The resulting plastic strain is mainly deviatoric in these samples. In the case of samples deformed upon larger external pressure, the difference with low pressure samples is not only the number of plastic events, but mainly the larger size and the larger amplitude of the plastic events. In this case, the densification is as important as the deviatoric strain.

\section{Local stress-strain behavior}

Is the mechanical response at a local scale reminiscent of the mechanical response at large scales? In Fig. 15, the local coarse-grained shear strain variation $\left(d \epsilon_{k}\right)$ is shown as a function of the amplitude of the plastic event. Equivalent shear strain $\left(\epsilon_{k}\right)$ is computed the same way as $k$ [in Eq. (1), replace the components of the stress tensor by the coarse-grained strain components]. The coarse-grained strain tensor $\underline{\varepsilon}(x, y, z)$ is computed from the coarse-grained displacement fiel $\overline{\bar{l}}$ d between each 10 steps as before. It can be seen in Fig. 15 that the relationship between the nonaffine displacement and local shear strain is linear. Therefore, if $u_{n a}$ is higher, the local strain increment is higher as well. The dashed line shows a linear regression between the two data. The slope of the line corresponds to the inverse of the elementary size of the local rearrangement,

$$
2 \varepsilon_{k}=\frac{u_{n a}}{2 r_{\mathrm{PE}}} .
$$

By taking $r_{\mathrm{PE}}=6.5 \AA$, a relatively good fit can be achieved, verifying the results already shown in Fig. 14(c).

Local stress tensors can also be calculated using coarse graining. The simulation provides us with the stress tensor on each atom using virial stresses [44]. With $5 \AA$ coarse-graining scale, the stresses are well defined and can be compared to strains. Figure 16 shows the evolution of the local load $k$ [Eq. (1)] and the amplitude of the nonaffine displacement field
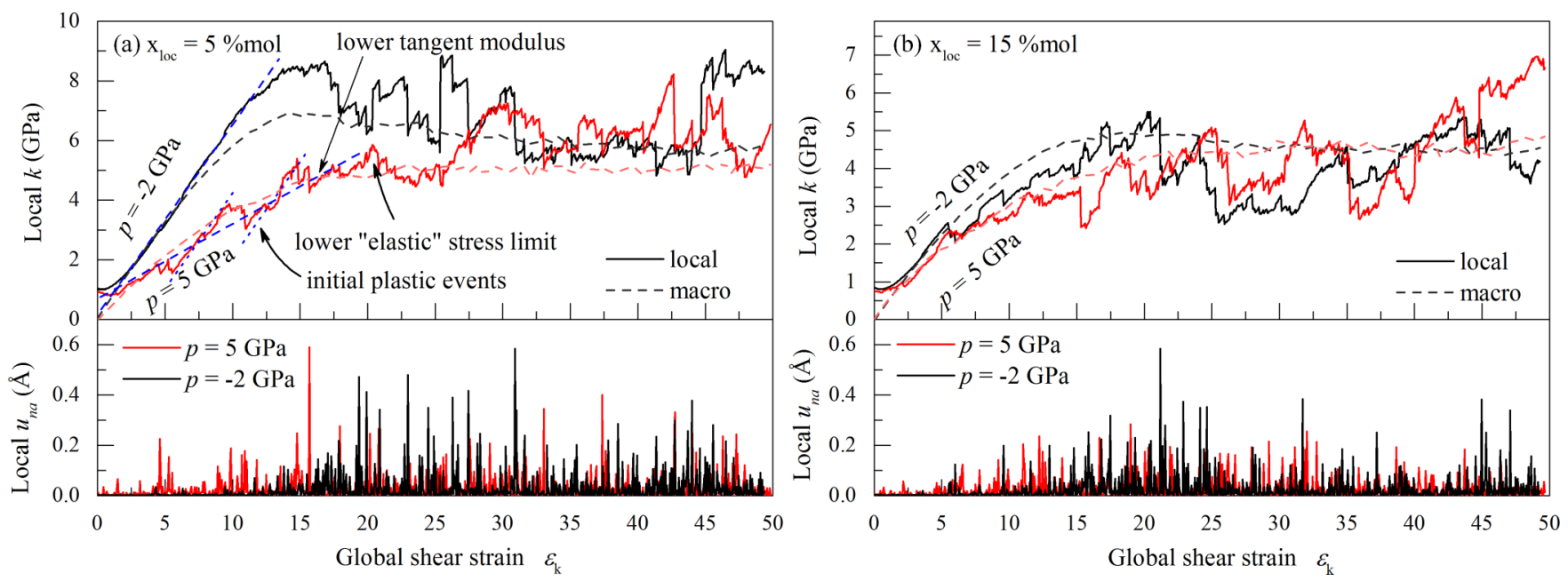

FIG. 16. Local equivalent shear stress $(k)$ as a function of global shear strain for two different local compositions taken from NSx5. With the dashed lines, the global stress-strain curves are plotted to visualize the similarities between the local and global behavior for different compositions. Under each diagram, the value of the nonaffine displacement field is plotted to show the comparison between stress drops and plastic events. 
at two different locations in sample NSx5 for $p=-2$ and $p=5 \mathrm{GPa}$. The chosen locations have significantly different compositions: in Fig. 16(a), the $\mathrm{Na}_{2} \mathrm{O}$ content is $5 \%$, and in Fig. 16(b), it is $15 \%$. In this way, two different stress responses can be observed inside the same sample. Using dashed lines, the large scale global stress curve is plotted for the same composition to highlight similarities between the global and local responses.

It can be seen in Fig. 16(a) that the maximum stress $\left(k_{\max }^{p=-2}=8.5 \mathrm{GPa}\right)$ at $p=-2 \mathrm{GPa}$ in the end of the elastic regime $\left(\epsilon_{x y}=15 \%\right)$ is higher than at $p=5 \mathrm{GPa}\left(k_{\max }^{p=5}=\right.$ $5.9 \mathrm{GPa})$. As can be deduced from the stress fluctuations along the stress-strain curves, the reason for the maximum stress decay is not the stiffness lowering with pressure (as shown in Fig. 6 at large scales), but the stress decreases due to the influence of initial PEs. Indeed, at larger pressures, the initial PEs are more frequent and with larger amplitudes. Therefore, we can conclude that the increase of the shear modulus observed at a large scale results from local plastic strain hardening. It can also be seen in Fig. 16(a) that compositions with low sodium content behave elastically, while the structure remains intact. If the system is compressed, the network is damaged and plasticity appears at earlier stages, making the material yield faster.

The same phenomenon is observed in Fig. 16(b) at sodium rich regions. Thanks to the mobility of the network modifiers, the materials area plastifies rapidly. In this way, it reduces the elastic maximum strength. Sodium also acts at negative pressure; therefore, the effect of pressure is much smaller in sodium rich regions.

Finally, in Fig. 17, the local maximum equivalent shear stress $\left(k_{\max }\right)$ is plotted as a function of the local composition. The plotted maximum stress or so-called strength value is computed by registering the maximum stress before the first large stress decrement ( $\delta k)$ (blue dotted line in Fig. 17 inset).

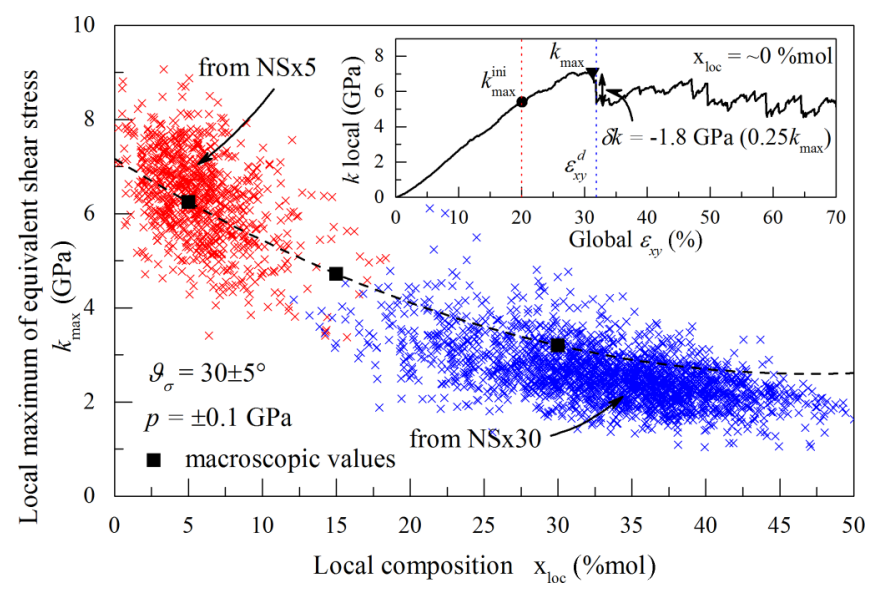

FIG. 17. Quasielastic local shear strength $\left(k_{\max }\right)$ at the end of the quasielastic regime as a function of local composition. The values plotted in the main figure are shown in the inset using a triangle. The results presented in the figure are taken at quasizero pressure $( \pm 0.1 \mathrm{GPa})$ and from the pure shear meridian in the Haigh-Westergaard space [47]. The inset shows the method which is used to determine the quasielastic shear strength from the stress curves; detailed discussion can be found in the text.
This stress release indicates that the elastic load bearing capacity of the sample is exceeded. Clear correlation is observed between the strength and the local composition of the material. Moreover, the local composition dependence of the local stress maxima is centered on the composition dependence of the global stress maximum, thus showing again the correspondence between the two.

\section{DISCUSSION AND CONCLUSION}

Molecular statics calculations were performed to investigate the plastic response of sodium silicate glasses. Correlation is made between the yield behavior observed at a large scale and atomic rearrangements. The samples were first pressurized, then shear deformation was applied at constant pressure. After relaxing both shear and pressure, the initial and the final simulation box were compared in order to determine the global permanent shear and volumetric strains. In parallel, the coarse-grained nonaffine displacement field was used to identify the position, the amplitude, and the size of the plastic events. The characteristics of these microscopic transformation zones were then used to explain the change in the global yield strength and the governing plastic strain.

Global results showed that with the addition of sodium, both hydrostatic tensile and shear yield strength are reduced. $\mathrm{Na}$ acts as a catalyst of plasticity, especially in the early stages of the deformation. The response thus transforms from a brittle softening to a ductile one for sufficiently large sodium content. Pressurization (as permanent volume loss) acts in a similar way: it reduces maximum shear stress and increases ductility. The increment of the sodium content helps the material to yield rapidly, reducing the size of the quasielastic domain in the stress space. The only difference between large sodium content and pressurized samples seems to be the relative amount of volumetric versus equivalent shear strain, and the absolute number of PEs.

Our results strengthen the connection between global and local plasticity, restricted to local events with finite extent. Clear spatial and statistical correlation was made between the plastic events identified by the nonaffine displacement field and the local strain field. The elementary diameter of the microscopic plastic rearrangements were found around $13 \AA$, which approximately corresponds to 6-8 interatomic distances. This is the typical size of plastic cores (also referred to as shear transformation zones, STZs) observed in other amorphous materials $[15,19,77]$. Using the statistically identified plastic events (from the nonaffine displacement field), three deformation stages were distinguished: an initial mainly elastic stage, a gradual plastification, and a stationary plastic plateau. By increasing the pressure, larger plastic events were observed and with larger amplitude, while in sodium rich samples, the number of (smaller) plastic events is two times higher. Both large number and large size of plastic events contribute to reduce the global yield stress [20]. In all cases, plastic rearrangements occur at sodium rich local regions, showing that the network modifier acts as a catalyst for plasticity. Local stress analysis showed that by pressurizing the samples, a large number of plastic events appear in the initial quasielastic stage, reducing the maximum elastic strength and increasing permanent deformation. Thus, increasing the 
pressure or the sodium content, the samples plastify in an earlier stage, becoming more ductile at a large scale.

Local analysis showed that $\mathrm{Na}$ is the most mobile ion in the structure. However, either pressure or the increment of composition reduces the amplitude of this motion, trapping sodium inside the silica network. This effect becomes significant in the plastic flow regime only. It would be interesting to compare this description to the local rigidity network described in Ref. [9].

Finally, excellent correlation was found between locally and globally measured maximum yield strength, thus confirming that plastic events identified using the nonaffine displacements are indeed responsible for the change in the elastoplastic behavior observed at large scales.

The size of the plastic events was shown to be pressure sensitive and thus without direct structural origin. The number of plastic events depends on the nucleation criteria that are related to local sodium content [35]. Sodium silicate glasses are thus examples of glasses where complex composition simplifies the research of local criteria due to the large mobility of sodium ions. However, the precise role of sodium at the different stages of the deformation is still under study and cannot be solved easily. In the plastic plateau, for example, the role of sodium is less evident, suggesting a self-organized flow behavior. It is the same for the distribution of eigenstrains and for the pressure sensitivity of the number and of the size of plastic events. Is there any collective effect in these characteristics? Among other perspectives, understanding nucleation criteria for plasticity at different load levels, to get a more quantitative analysis of the local plasticity in amorphous materials and to open the door for definitely more quantitative prediction of plastic deformation, is still a challenging task. A realistic description needs to introduce finite temperature effects as well.

\section{ACKNOWLEDGMENTS}

The authors thank G. Kermouche, E. Barthel, and T. Albaret for motivating discussions, and B. Champagnon, T. Rouxel, V. Martinez, and C. Martinet for sharing experimental results. This work was supported by the French Research National Agency program MultiSil (Grant No. ANR-13-BS09-0012).
[1] J. Barton and C. Guillemet, Le Verre : Science et Technologie (EDP Sciences, 2005).

[2] R. Brückner, J. Non-Crystal. Solids 5, 123 (1970).

[3] S. Romeis, J. Paul, P. Herre, D. de Ligny, J. Schmidt, and W. Peukert, Scr. Mater. 108, 84 (2015).

[4] K. W. Peter, J. Non-Crystal. Solids 5, 103 (1970).

[5] C. R. Kurkjian, G. W. Kammlott, and M. M. Chaudhri, J. Amer. Ceramic Soc. 78, 737 (1995).

[6] H. Ji, V. Keryvin, T. Rouxel, and T. Hammoudab, Scr. Mater. 55, 1159 (2006).

[7] T. Deschamps, C. Martinet, J. L. Bruneel, and B. Champagnon, J. Phys.: Condens. Matter 23, 035402 (2011).

[8] C. Hermansen, J. Matsuoka, S. Yoshida, H. Yamazaki, Y. Kato, and Y. Yue, J. Non-Crystal. Solids 364, 40 (2013).

[9] B. Wang, Y. Yu, M. Wang, J. C. Mauro, and M. Bauchy, Phys. Rev. B 93, 064202 (2016).

[10] W. T. Read, Dislocations In Crystals (McGraw Hill, New York, 1953).

[11] F. Spaepen, Acta Metal. 25, 407 (1977).

[12] A.-S. Argon, Acta Metal. 27, 47 (1979).

[13] J. D. Eshelby, Proc. R. Soc. London 241, 376 (1957).

[14] C. Maloney and A. Lemaître, Phys. Rev. Lett. 93, 016001 (2004).

[15] A. Tanguy, F. Leonforte, and J.-L. Barrat, Europhys. J. E 20, 355 (2006).

[16] R. Dasgupta, H. G. E. Hentschel, and I. Procaccia, Phys. Rev. Lett. 109, 255502 (2012).

[17] F. Puosi, J. Rottler, and J.-L. Barrat, Phys. Rev. E 89, 042302 (2014).

[18] A. Nicolas, F. Puosi, H. Mizuno, and J.-L. Barrat, J. Mech. Phys. Solids 78, 333 (2015).

[19] T. Albaret, A. Tanguy, F. Boioli, and D. Rodney, Phys. Rev. E 93, 053002 (2016).

[20] C. Fusco, T. Albaret, and A. Tanguy, Phys. Rev. E 82, 066116 (2010).

[21] A. Amon, V.B. Nguyen, A. Bruand, J. Crassous, and E. Clément, Phys. Rev. Lett. 108, 135502 (2012).
[22] P. Marmottant and F. Graner, Soft Matter 9, 9602 (2013).

[23] C. Fusco, T. Albaret, and A. Tanguy, Europhys. J. E 37, 43 (2014).

[24] P. Sollich, F. Lequeux, P. Hebraud, and M. E. Cates, Phys. Rev. Lett. 78, 2020 (1997).

[25] M. Talamali, V. Petäjä, D. Vandembroucq, and S. Roux, Phys. Rev. E 84, 016115 (2011).

[26] M. Tsamados, A. Tanguy, C. Goldenberg, and J. L. Barrat, Phys. Rev. E 80, 026112 (2009).

[27] D. Rodney, A. Tanguy, and D. Vandembroucq, Model. Simul. Mater. Sci. Eng. 19, 083001 (2011).

[28] J. Perepezko, S. Imhoff, M. Chen, J. Wang, and S. Gouzales, Proc. Nat. Acad. Sci. USA 111, 3938 (2014).

[29] G. Kermouche, E. Barthel, D. Vandembroucq, and P. Dubujet, Acta Mater. 56, 3222 (2008).

[30] G. Molnár, P. Ganster, A. Tanguy, E. Barthel, and G. Kermouche, Acta Mater. 111, 129 (2016).

[31] B. Mantisi, G. Kermouche, E. Barthel, and A. Tanguy, Phys. Rev. E 93, 033001 (2016).

[32] B. W. H. van Beest, G. J. Kramer, and R. A. van Santen, Phys. Rev. Lett. 64, 1955 (1990).

[33] X. Yuan and A. N. Cormack, J. Non-Crystal. Solids 283, 69 (2001).

[34] B. Mantisi, A. Tanguy, G. Kermouche, and E. Barthel, Europhys. J. B 85, 304 (2012).

[35] G. Molnár, P. Ganster, J. Török, and A. Tanguy, J. Non-Crystal. Solids 440, 12 (2016).

[36] S. Plimpton, J. Comput. Phys. 117, 1 (1995).

[37] A. Tanguy, JOM 67, 1832 (2015).

[38] M. Fábián, P. Jóvári, E. Sváb, G. Mészáros, T. Proffen, and E. Veress, J. Phys.: Condens. Matter 19, 335209 (2007).

[39] H. Maekawa, T. Maekawa, K. Kawamura, and T. Yokokawa, J. Non-Crystal. Solids 127, 53 (1991).

[40] T. Charpentier, S. Ispas, M. Profeta, F. Mauri, and C. J. Pickard, J Phys. Chem. B 108, 4147 (2004). 
[41] D. Theodorouand and U. W. Suter, Macromolecules 19, 139 (1986).

[42] C. A. Schuh and A. C. Lund, Nat. Mater. 2, 449 (2003).

[43] A. Nicolas and J. L. Barrat and J. Rottler, Phys. Rev. Lett. 116, 058303 (2016).

[44] A. P. Thompson, S. J. Plimpton, and W. Mattson, J. Chem. Phys. 131, 154107 (2009).

[45] Furthermore, it is important to note that during just the constant pressure calculation, we do not necessarily demand equal axial stresses, e.g., $\sigma_{x x}=1, \sigma_{y y}=0, \sigma_{z z}=-1$ results in $p=0$, although the stress state is not on the hydrostatic axis and consequently unwanted deviatoric stress is observed. In order to avoid this case, a constant hydrostatic stress state is required.

[46] R. von Mises, Nachr. Ges. Wiss. Goettingen, Math. Phys. Kl. 1913, 582 (1913).

[47] M.-H. Yu, Generalized Plasticity (Springer Science and Business Media, New York, 2006).

[48] H. Hibbitt, P. Marcal, and J. Rice, Intl. J. Solids Struct. 6, 1069 (1970).

[49] Z.P. Bazant, Intl. J. Solids Struct. 33, 2887 (1996).

[50] C. Meade and R. Jeanloz, Phys. Rev. B 35, 236 (1987).

[51] T. Sato and N. Funamori, Phys. Rev. Lett. 101, 255502 (2008).

[52] G. Molnar, P. Ganster, A. Tanguy, J. Torok, and G. Kermouche, MRS Adv. 1, 1797 (2016).

[53] $p-\epsilon_{V}$ curves are fitted with a fifth-order polynomial function, then the derivative of the fit is taken with respect to the volume change.

[54] T. Rouxel, H. Ji, J. P. Guin, F. Augereau, and B. Rufflé, J. Appl. Phys. 107, 094903 (2010).

[55] T. Deschamps, A. Kassir-Bodon, C. Sonneville, J. Margueritat, C. Martinet, D. de Ligny, A. Mermet, and B. Champagnon, J. Phys.: Condens. Matter 25, 025402 (2013).

[56] Q. Zhao, M. Guerette, G. Scannell, and L. Huang, J. NonCrystal. Solids 358, 3418 (2012).
[57] P. W. Bridgman, Amer. J. Sci. 10, 359 (1925).

[58] M. Grimsditch, Phys. Rev. Lett. 52, 2379 (1984).

[59] T. Deschamps, J. Margueritat, C. Martinet, A. Mermet, and B. Champagnon, Sci. Rep. 4, 7193 (2014).

[60] D. Wakabayashi, N. Funamori, T. Sato, and T. Taniguchi, Phys. Rev. B 84, 144103 (2011).

[61] C. A. Coulomb, Mem. Acad. Roy. Div. Sav. 7, 343 (1776).

[62] D. C. Drucker and W. Prager, Quarter. Appl. Math. 10, 157 (1952).

[63] A. C. Lund and C. A. Schuh, Acta Mater. 53, 3193 (2005).

[64] In this way, all strains presented have the magnitude of the normal strains.

[65] $\gamma_{x y}=2 \epsilon_{x y}$ is called the engineering strain.

[66] J. D. Mackenzie, J. Amer. Ceramic Soc. 46, 461 (1963).

[67] T. M. Gross and M. Tomozawa, J. Appl. Phys. 104, 063529 (2008).

[68] A. Tanguy, J. P. Wittmer, F. Leonforte, and J.-L. Barrat, Phys. Rev. B 66, 174205 (2002).

[69] D. Weaire and J. P. Kermode, Philos. Mag. B 50, 379 (1984).

[70] C. Goldenberg, A. Tanguy, and J.-L. Barrat, Europhys. Lett. 80, 16003 (2007)

[71] P. Jund, E. Sunyer, and R. Jullien, J. Non-Crystal. Solids 352, 5188 (2006)

[72] M. Rarivomanantsoa, P. Jund, and R. Jullien, J. Chem. Phys. 120, 4915 (2004).

[73] M. Bauchy, B. Guillot, M. Micoulaut, and N. Sator, Chem. Geol. 346, 47 (2013).

[74] M. L. Falk and J.-S. Langer, Phys. Rev. E 57, 7192 (1998).

[75] E. Homer, Acta Mater. 63, 44 (2014).

[76] G. P. Shrivastav, P. Chaudhuri, and J. Horbach, Phys. Rev. E 94, 042605 (2016).

[77] Y. M. Beltukov, C. Fusco, D. A. Parshin, and A. Tanguy, Phys. Rev. E 93, 023006 (2016). 